\title{
Étude anthropologique des vestiges humains récemment découverts issus de la couche 2 de l'abri Pataud (Les Eyzies-de-Tayac-Sireuil, Dordogne, France)
}

\author{
Anthropological study of recently discovered human remains from the layer 2 of the abri Pataud \\ (Les Eyzies-de-Tayac-Sireuil, Dordogne, France)
}

\author{
S. Villotte $\cdot$ L. Chiotti $\cdot$ R. Nespoulet $\cdot$ D. Henry-Gambier \\ Reçu le 3 mars 2015; accepté le 4 mai 2015 \\ (C) Société d'anthropologie de Paris et Lavoisier SAS 2015
}

Résumé L'abri Pataud, situé dans la commune des Eyziesde-Tayac-Sireuil (Dordogne, France), a été fouillé dans les années cinquante et soixante et fait l'objet de nouvelles fouilles depuis 2005. La couche 2 a livré plus de 400 restes humains, les seuls connus pour le Gravettien final européen. Cet article présente les caractéristiques morphologiques de 62 vestiges humains découverts récemment. Ces vestiges s'inscrivent dans la variabilité morphologique gravettienne et ne présentent pas de caractéristiques notables, mis à part, pour les restes crâniens, la présence de nombreux foramens surnuméraires et celle de cuspides surnuméraires. L'étude morphologique et métrique permet en outre d'associer certains ossements aux individus adultes P1 et P5.

Mots clés Paléolithique supérieur · Gravettien ·

Paléoanthropologie

\begin{abstract}
The Abri Pataud, located in the village of Les Eyzies-de-Tayac-Sireuil (Dordogne, France), was excavated in the 1950s and 1960s, and new excavations have been carried out since 2005 . More than 400 human remains, the only
\end{abstract}

S. Villotte $(\bowtie) \cdot$ D. Henry-Gambier

CNRS, Univ. Bordeaux, MCC, PACEA,

UMR5199. Bât. B8. Allée Geoffroy Saint-Hilaire - CS 50023,

F-33615 Pessac, France

e-mail : s.villotte@pacea.u-bordeaux1.fr

L. Chiotti

Département de Préhistoire,

Muséum national d'Histoire naturelle, UMR 7194 CNRS,

Abri Pataud, 20, rue du Moyen-âge,

24620 Les Eyzies-de-Tayac-Sireuil

R. Nespoulet

Département de Préhistoire,

Muséum national d'Histoire naturelle, UMR 7194 CNRS,

Musée de l'Homme, 17, place du Trocadéro, 75116 Paris examples known for the Final Gravettian in Europe, were found in the layer 2 . This paper presents the morphological characteristics of 62 recently discovered human remains. These remains fall well within the Gravettian variability known to date and display no notable features, except the presence of supernumerary foramina on some cranial bones and supernumerary cusps on the teeth. Moreover, this study enabled several new elements to be assigned to adult individuals P1 and P5.

Keywords Upper Paleolithic · Gravettian ·

Paleoanthropology

\section{Introduction}

L'abri Pataud, situé dans la commune des Eyziesde-Tayac-Sireuil (Dordogne, France), a été fouillé entre 1958 et 1964 par Movius et fait l'objet depuis 2005 de nouvelles fouilles dirigées par Nespoulet et Chiotti [1]. L'importante stratigraphie a livré 14 niveaux principaux d'occupation, attribués à l'Aurignacien, au Gravettien et au Solutréen.

La couche 2, fouillée en 1958 puis en 1963, est attribuée au Gravettien final et située dans un intervalle chronologique de 28000-26000 cal. BP [1,2]. Depuis 2005, de nouvelles fouilles de cette couche ont été entreprises au Nord de l'abri, dans un secteur directement adjacent aux fouilles Movius [1].

La couche 2 a livré des milliers de vestiges archéologiques qui relèvent essentiellement de la sphère d'habitat $[3,4]$. Toutefois, le fond de l'abri, où la hauteur sous voûte était inférieure à un mètre, a vraisemblablement été peu propice à l'habitat et semble plutôt avoir été un espace réservé à des activités à caractère rituel et au dépôt des morts [3-7]. Cette couche a livré plus de 400 restes humains, les seuls connus pour le Gravettien final français [6], tous concentrés en fond 
d'abri. Les caractéristiques morphologiques des vestiges humains identifiés durant les fouilles Movius ont été présentées par Billy [8] et Legoux [9-11]. Les restes humains découverts plus récemment, lors des nouvelles fouilles ou durant le tri de la faune des fouilles Movius et des déblais d'une fouille clandestine, n'ont quant à eux fait l'objet que d'une description biologique partielle [6]. L'objectif de cet article est de présenter en détail ces vestiges.

\section{Matériel et méthodes}

Quatre cent vingt-neuf dents isolées ou vestiges osseux humains ont été reconnus pour la couche 2 [6]. Chacun de ces vestiges dispose maintenant d'un numéro unique, attribué depuis la reprise des fouilles en 2005 .

Ces vestiges appartiennent à au moins six individus, 2 adultes féminins, un adulte masculin, et 3 sujets immatures $[6,12]$ :

- P1 est une femme décédée entre 20 et 29 ans, essentiellement représentée par la tête osseuse complète, un os coxal gauche, de nombreuses côtes et vertèbres ainsi que la plupart des os des mains et des pieds.

- P2 est un enfant d'âge dentaire d'environ 5 mois représenté par une mandibule, des dents isolées et une partie du squelette infracrânien.

- P3 est une femme identifiée par des os des membres supérieurs en connexion.

- P4 est un enfant d'âge dentaire d'environ 8 mois représenté par le neurocrâne complet, un fragment de mandibule, des dents isolées et une partie du squelette infracrânien.

- P5 est un homme d'âge inconnu représenté par un humérus, un radius et une ulna gauches.

- P6 est un sujet immature dont l'âge dentaire se situe entre 4 et 7 ans, identifié par des fragments de crâne, des dents isolées et un métacarpien.

D'autres vestiges d'adultes n'ont pu être attribués avec certitude à l'un de ces sujets, un numéro particulier (PX1, $\mathrm{PX} 2$, etc.) a donc été affecté à chacun d'entre eux [6].

Parmi les 429 vestiges humains reconnus, 62 ont été découverts récemment : lors du tri de la faune des fouilles Movius $(\mathrm{N}=22)$, du tri des déblais de la fouille clandestine $(\mathrm{N}=36)$, et des nouvelles fouilles $(\mathrm{N}=3)$ [6] (Tableau 1).

Les critères d'attribution de ces vestiges à chacun des sujets identifiés ont déjà fait l'objet d'une présentation détaillée [6]. Les résultats des analyses morphologiques (observations macroscopiques et à la loupe binoculaire) et métriques présentés ici permettent cependant de compléter ces attributions.

Les mesures indiquées par un « $M$ » sont définies par Martin et présentées dans Braüer [13]. Les autres mesures sont définies dans Sládek et al. [14]. Les mesures entre parenthèses sont estimées. Toutes les valeurs sont en millimètres. Lorsque cela semblait pertinent, les mesures ont été comparées à celles des autres vestiges de l'abri Pataud conservés au Musée de l'Homme et celles des sujets du Gravettien (Tableaux 2, 3). Dans les tableaux, les mesures et les données statistiques de comparaison (moyennes et écartstypes) sont indiquées en italique. L'usure dentaire a été enregistrée selon Smith [15] et les caractères discrets dentaires selon Turner et al [16].

\section{Résultats}

Les 62 vestiges sont présentés par individu et par logique anatomique.

\section{Vestiges attribués à $\mathbf{P 1}$}

\section{Vestiges osseux crâniens}

\section{Os hyoüde (AP/89-2-37968)}

Cet élément correspond au corps d'un os hyoïde totalement ossifié (Fig. 1a), avec une légère altération des marges (dimension maximale : 18,5 mm). En dépit de l'érosion des marges, une majorité des surfaces articulaires pour les grandes cornes est préservée, indiquant que celles-ci n'étaient pas soudées. Le corps présente une forme plutôt ovalaire. Les zones d'insertion du muscle génio-hyoïdien sont très creusées et la crête cruciforme est très saillante supérieurement, formant une petite épine. La concavité postérieure est très marquée. Avec un diamètre transverse de $18,5 \mathrm{~mm}$, une hauteur au milieu de $8,9 \mathrm{~mm}$, et une épaisseur antéro-postérieure de $6,2 \mathrm{~mm}$, cet os est de très petites dimensions [cf. 30].

\section{Dents}

Onze dents permanentes (Fig. 2) ont été retrouvées dans les déblais de la fouille clandestine, qui a concerné les zones $\mathrm{F} /$ GVIII. Ces dents s'insèrent parfaitement (du moins pour les sept dont la racine n'est pas abimée) dans les alvéoles des maxillaires et de la mandibule de P1. Cela conforte l'hypothèse d'un prélèvement par les gravettiens de la tête osseuse et de son dépôt trois mètres plus loin, dans la zone FV/VI [6]. Les mesures sont présentées dans le tableau 4. 
Tableau 1 Inventaire des vestiges. FC : fouille clandestine, TF : tri de faune (réalisé en 1998 par T.-S. Cho et repris en 2006-2008 par D. Henry-Gambier, C. Beauval et S. Villotte), FR : fouilles récentes / Inventory of the human remains. FC: illicit excavations, TF: found in the faunal remains (research carried out in 1998 by T.-S. Cho and in 2006-2008 by par D. Henry-Gambier, C. Beauval, and S. Villotte), FR: new excavations.

\begin{tabular}{|c|c|c|c|c|}
\hline $\mathbf{N}^{\circ}$ & Vestiges & Attribution & Remarques & Origine \\
\hline AP/89-2-37968 & Hyoïde & $\mathrm{P} 1$ & & $\mathrm{FC}$ \\
\hline $\mathrm{AP} / 89-2-62$ & Incisive 1 sup. droite & $\mathrm{P} 1$ & & $\mathrm{FC}$ \\
\hline $\mathrm{AP} / 89-2-71$ & Incisive 1 sup. gauche & $\mathrm{P} 1$ & Contiguïté articulaire & $\mathrm{FC}$ \\
\hline $\mathrm{AP} / 89-2-66$ & Incisive 2 sup. droite & P1 & Contiguïté articulaire & $\mathrm{FC}$ \\
\hline $\mathrm{AP} / 89-2-61$ & Canine sup. droite & $\mathrm{P} 1$ & & $\mathrm{FC}$ \\
\hline AP/89-2-68 & Canine sup. gauche & $\mathrm{P} 1$ & Contiguïté articulaire & $\mathrm{FC}$ \\
\hline $\mathrm{AP} / 89-2-67$ & Incisive 1 ou 2 inf. & $\mathrm{P} 1$ & Contiguïté articulaire possible & $\mathrm{FC}$ \\
\hline $\mathrm{AP} / 89-2-63$ & Incisive 1 ou 2 inf. & $\mathrm{P} 1$ & Contiguïté articulaire possible & $\mathrm{FC}$ \\
\hline $\mathrm{AP} / 89-2-64$ & Canine inf. droite & $\mathrm{P} 1$ & Contiguïté articulaire & $\mathrm{FC}$ \\
\hline $\mathrm{AP} / 89-2-70$ & Canine inf. gauche & P1 & & $\mathrm{FC}$ \\
\hline $\mathrm{AP} / 89-2-69$ & Prémolaire 2 inf. droite & $\mathrm{P} 1$ & & $\mathrm{FC}$ \\
\hline $\mathrm{AP} / 89-2-65$ & Prémolaire 2 inf. gauche & $\mathrm{P} 1$ & Contiguïté articulaire & $\mathrm{FC}$ \\
\hline $\mathrm{AP} / 89-2-309$ & Vertèbre Cervicale 1 & P1 & Contiguïté articulaire & $\mathrm{FC}$ \\
\hline AP/89-2-37979 & Vertèbre Cervicale 5 ou 6 & $\mathrm{P} 1$ & & $\mathrm{FC}$ \\
\hline AP/89-2-37975 & $\begin{array}{l}\text { Vertèbre Cervicale } \\
\text { ou Thoracique }\end{array}$ & $\mathrm{P} 1$ & & $\mathrm{FC}$ \\
\hline $\mathrm{AP} / 89-2-288$ & Vertèbre Thoracique & $\mathrm{P} 1$ & Détruite & $\mathrm{FC}$ \\
\hline AP/89-2-37976 & Vertèbre Thoracique & $\mathrm{P} 1$ & & $\mathrm{FC}$ \\
\hline AP/89-2-37967 & Vertèbre Thoracique & $\mathrm{P} 1$ & & $\mathrm{FC}$ \\
\hline $\mathrm{AP} / 89-2-37978$ & Vertèbre Thoracique & $\mathrm{P} 1$ & & FC \\
\hline AP/89-2-37980 & Vertèbre Thoracique & $\mathrm{P} 1$ & & $\mathrm{FC}$ \\
\hline $\mathrm{AP} / 89-2-313$ & Vertèbre Thoracique & $\mathrm{P} 1$ & & $\mathrm{FC}$ \\
\hline AP/89-2-37974 & Vertèbre Thoracique Haute & P1 & & $\mathrm{FC}$ \\
\hline $\mathrm{AP} / 89-2-37977$ & Vertèbre Thoracique $11 ?$ & $\mathrm{P} 1$ & Contiguïté articulaire & $\mathrm{FC}$ \\
\hline $\mathrm{AP} / 89-2-310$ & Vertèbre Thoracique 12 ? & $\mathrm{P} 1$ & & $\mathrm{FC}$ \\
\hline AP/89-2-37973 & Côte droite 1 & $\mathrm{P} 1$ & & $\mathrm{FC}$ \\
\hline AP/89-2-299 & Côte gauche 12 & $\mathrm{P} 1$ & & $\mathrm{FC}$ \\
\hline $\mathrm{AP} / 63-2-37960$ & Scaphoïde droit & P1 & Symétrie & TF (2006) \\
\hline AP/89-2-37970 & Trapézoïde droit & $\mathrm{P} 1$ & Contiguïté articulaire & $\mathrm{FC}$ \\
\hline AP/89-2-37971 & MTC gauche ? 4 ? & P1 & & $\mathrm{FC}$ \\
\hline AP/89-2-312 & MTC droit $5 ?$ & $\mathrm{P} 1$ & & $\mathrm{FC}$ \\
\hline $\mathrm{AP} / 89-2-306$ & MTC gauche 4 & P1 & Symétrie probable & $\mathrm{FC}$ \\
\hline $\mathrm{AP} / 89-2-465$ & Phalange dist. 5 Main & $\mathrm{P} 1$ & Symétrie & $\mathrm{FC}$ \\
\hline $\mathrm{AP} / 63-1 / 2-37962$ & Phalange méd. gauche 5 Main & $\mathrm{P} 1$ & $\mathrm{~Pb}$. de dimension & TF (2006) \\
\hline AP/89-2-302 & Phalange prox. gauche 4 Main & $\mathrm{P} 1$ & Symétrie & $\mathrm{FC}$ \\
\hline $\mathrm{AP} / 89-2-37972$ & MTT droit $4 ?$ & $\mathrm{P} 1$ & Symétrie probable & $\mathrm{FC}$ \\
\hline $\mathrm{AP} / 10-2-\mathrm{W} 74 \mathrm{~A}-1$ & $\begin{array}{l}\text { Incisive déciduale } 2 \text { sup. } \\
\text { gauche }\end{array}$ & $\mathrm{P} 2$ & & FR \\
\hline $\mathrm{AP} / 89-2-72$ & $\begin{array}{l}\text { Molaire déciduale } 2 \text { sup. } \\
\text { gauche }\end{array}$ & $\mathrm{P} 2$ & & TF (1998) \\
\hline $\mathrm{AP} / 08-2-\mathrm{W} 76 \mathrm{C}-140$ & Vertèbre Lombaire & $\mathrm{P} 2$ & & FR \\
\hline AP/89-2-293 & Humérus droit & $\mathrm{P} 2$ & & TF (1998) \\
\hline $\mathrm{AP} / 58-2-37955$ & Humérus gauche & $\mathrm{P} 2$ & & TF (2006) \\
\hline $\mathrm{AP} / 63-2-37961$ & Coxal gauche? & $\mathrm{P} 2$ & Très gros par rapport à l'ischium & TF (2006) \\
\hline AP/89-2-37969 & Fémur droit & $\mathrm{P} 2$ & Symétrie & TF (1998) \\
\hline $\mathrm{AP} / 63-2-37953$ & Tibia droit & $\mathrm{P} 2$ & Symétrie probable & (Suite page suivante) \\
\hline
\end{tabular}




\begin{tabular}{|c|c|c|c|c|}
\hline $\mathbf{N}^{\circ}$ & Vestiges & Attribution & Remarques & Origine \\
\hline $\mathrm{AP} / 58-2-\mathrm{F} 2283$ & Canine déciduale sup. droit & P6 & Contiguïté articulaire & TF (1998) \\
\hline AP/07-2-W76A-T550 & $\begin{array}{l}\text { Incisive déciduale } 2 \text { sup. } \\
\text { gauche }\end{array}$ & P6 & & FR \\
\hline $\mathrm{AP} / 58-2-37956$ & Vertèbre Cervicale 1 & P6 & & TF (2006) \\
\hline $\mathrm{AP} / 58-2-37957$ & Vertèbre Cervicale 2 & P6 & & TF (2006) \\
\hline $\mathrm{AP} / 58-2-37959$ & Clavicule gauche & P6 & & TF (2006) \\
\hline $\mathrm{AP} / 58-2-37952$ & Scapula droite & P6 & & TF (2006) \\
\hline $\mathrm{AP} / 58-2-2 \mathrm{~F} 592$ & Scapula gauche & PX13 & P5 ? Aspect, dimensions & TF (1998) \\
\hline $\mathrm{AP} / 58-2-\mathrm{F} 1026 \mathrm{~A}$ & Clavicule gauche & PX14 & P5 ? Doublon, aspect, dimensions & TF (1998) \\
\hline $\mathrm{AP} / 58-2-\mathrm{F} 1026 \mathrm{~B}$ & Clavicule droite & PX15 & P5 ? Doublon, aspect, dimensions & TF (1998) \\
\hline $\mathrm{AP} / 58-2-2 \mathrm{~F} 1011$ & Fémur droit & PX16 & P1 ? dimensions, ocre & TF (1998) \\
\hline $\mathrm{AP} / 58-2-37966$ & Vertèbre Cervicale 2 & PX17 & & TF (2006) \\
\hline $\mathrm{AP} / 58-2-37958$ & Vertèbre Thoracique haute & PX18 & & TF (2006) \\
\hline $\mathrm{AP} / 58-2-37963$ & Temporal gauche & PX19 & & TF (2006) \\
\hline $\mathrm{AP} / 58-2-37954$ & Zygomatique droit & PX20 & & TF (2006) \\
\hline AP/--37801 & MTT gauche 1 & PX21 & & TF (1998) \\
\hline $\mathrm{AP} / 58-2-37802$ & Côte droite & PX22 & & TF (1998) \\
\hline $\mathrm{AP} / 58-2-37803$ & Côte & PX23 & & TF (1998) \\
\hline $\mathrm{AP} / 58-2-37804$ & Côte & PX24 & & TF (1998) \\
\hline $\mathrm{AP} / 58-2-37964$ & MTC droit 3 & PX31 & P5 ? Doublon, aspect, dimensions & TF (2006) \\
\hline
\end{tabular}

Tableau 2 Échantillon de comparaison pour les mesures infracrâniennes / Comparative sample for infracranial measurements.

\begin{tabular}{|llll|}
\hline Sujets masculins & Références & Sujets féminins & Références \\
\hline Baousso da Torre 1 et 2 & SV Obs. pers. & Abri Pataud P1 et P3 & DHG et SV Obs. pers. \\
Barma Grande 2 & {$[17]$ et Holliday com. pers. } & Barma di Caviglione 1 & Brǔžek et Trinkaus com. pers. \\
Cro Magnon 1 & {$[18,19] ;$ DHG et SV Obs. pers. } & Dolní Věstonice 3 & {$[14,20]$} \\
Dolní Věstonice 13 et 16 & {$[14,20]$} & Grotte des Enfants 5 & {$[21] ;$ DHG et SV Obs. pers. } \\
Grotte des Enfants 4 & {$[21] ;$ DHG et SV Obs. pers. } & Paglicci 25 & {$[22]$} \\
Paviland 1 & {$[23]$} & Veneri Parabita 2 & {$[24]$} \\
Sunghir 1 & {$[25]$} & & \\
Veneri Parabita 1 & {$[24]$} & & \\
\hline
\end{tabular}

\section{Incisives supérieures médiales droite (AP/89-2-62) et gauche (AP/89-2-71)}

AP/89-2-62 est complète mais l'apex de la racine est endommagée (dimension maximale : 20,6 mm), et une fissure longitudinale traverse la face vestibulaire de la couronne. AP/89-2-71 est également complète (dimension maximale : $19,6 \mathrm{~mm}$ ) mais toute la surface de la dent est très altérée et a été consolidée. De plus, la couronne est affectée par une fissure longitudinale ouverte. AP/89-2-62 s'articule assez bien avec le bloc craniofacial de $\mathrm{P} 1$; quant à AP/89-2-71, elle s'articule parfaitement.

Une plage de tarte est visible sur la face vestibulaire de AP/89-2-62. La face linguale des deux dents présente une surface polie et peu modelée : les crêtes mésiale et distale sont à peine discernables et le tubercule basal est effacé. L'usure est modérée (stade 4).

\section{Incisive supérieure latérale droite (AP/89-2-66)}

Cette dent est complète (dimension maximale : 20,9 mm). La racine est totalement calcifiée et l'apex est légèrement érodé. Cette dent s'articule bien avec le bloc craniofacial de P1.

Le bord occlusal de la couronne est modérément usé en oblique (stade 3). La face vestibulaire présente une série d'altération de l'émail dont une plus importante constitue une plage sensiblement circulaire masquée en partie par du tarte ou du sédiment. Il est impossible de se prononcer sur 


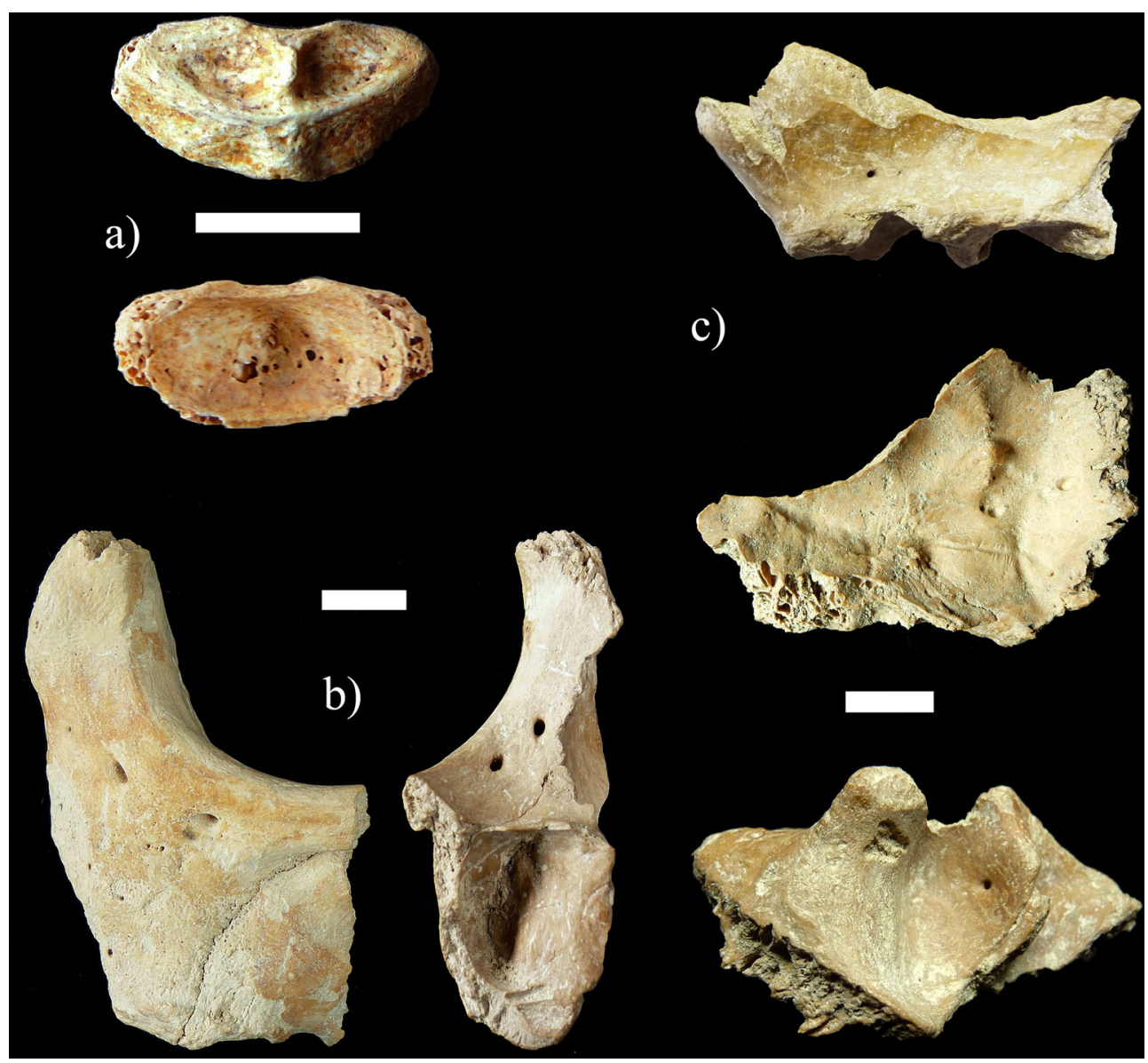

Fig. 1 Vestiges crâniens adultes (échelles : $1 \mathrm{~cm}$ ). a : os hyoïde (AP/89-2-37968), vues antérieure et postérieure, b : os zygomatique et maxillaire droits (AP/58-2-37954), vues antérieure et médiale, c : os temporal gauche (AP/58-2-37963), vues latérale, supéro-médiale, et inférieure (clichés S. Villotte) / Adult cranial remains (scales: $1 \mathrm{~cm}$ ). a: hyoid bone (AP/89-2-37968), anterior and posterior views, b: right zygomatic and maxillary bones (AP/58-2-37954), anterior and medial views, c: left temporal bone (AP/58-2-37963), lateral, supero-medial, and inferior views (images: $S$. Villotte).

l'étiologie : lésion, défaut de formation de l'émail ou altération d'origine taphonomique. La face linguale ne présente pas de relief particulier.

\section{Canines supérieures gauche (AP/89-2-68) et droite (AP/89-2-61)}

AP/89-2-68 est complète et totalement calcifiée (dimension maximale : 25,7 mm). La racine unique a un apex fermé. Sa surface est légèrement altérée. Cette dent remonte parfaitement avec le bloc craniofacial de P1. AP/89-2-62 est également complète mais très endommagée et consolidée (dimension maximale : $26,4 \mathrm{~mm}$ ).

Les deux dents sont très similaires. La pointe de la cuspide est usée, la dentine n'est pas dégagée à gauche (stade 2), alors qu'elle l'est à droite (stage 3). Une ligne de tarte $(<1 \mathrm{~mm}$ de largeur) se développe sur la face vestibulaire à
$2 \mathrm{~mm}$ du collet. Un tuberculum dentale bien dégagé est visible (stade 3) à gauche et à droite.

\section{Incisives inférieures (AP/89-2-63 et AP/89-2-67)}

AP/89-2-63 est complète et totalement calcifiée avec un apex fermé (dimension maximale : 20,8 $\mathrm{mm}$ ). La couronne de AP/89-2-67 est complète mais la racine est réduite de moitié par une cassure récente (dimension maximale : 13,7 mm).

Les deux dents sont symétriques et ont les mêmes caractéristiques. Leurs faces vestibulaire et linguale présentent un léger dépôt de tarte. Leur bord incisif est modérément usé horizontalement (stade 4). Leur face linguale ne présente aucun relief particulier. On devine sur les bords mésial et distal de AP/89-2-63, moins consolidée que sa symétrique, des facettes de contact.

Il parait très difficile d'identifier formellement ces dents. La morphologie évoque plus des incisives latérales (AP/89- 


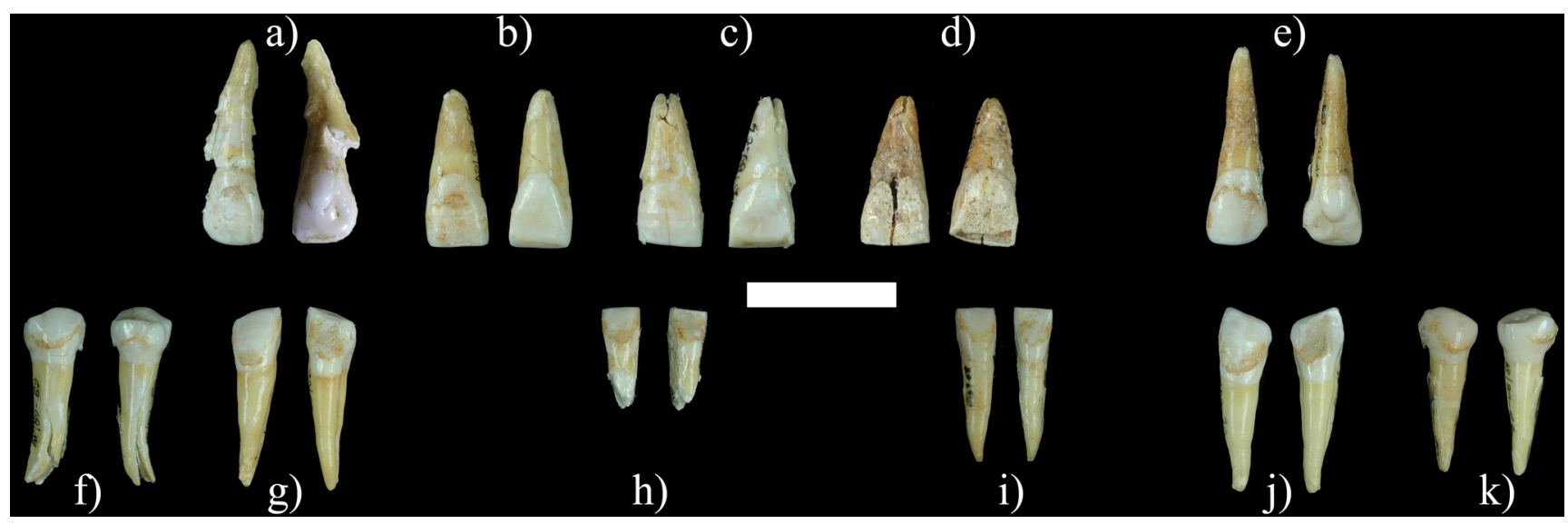

Fig. 2 Dents permanentes attribuées à P1 (échelle : $2 \mathrm{~cm}$ ), faces vestibulaire et linguale. a et e : canines supérieures droite (AP/89-2-61) et gauche (AP/89-2-68), b : incisive supérieure latérale droite (AP/89-2-66), c et d : incisives supérieures centrales droite (AP/89-2-62) et gauche (AP/89-2-71), $\mathrm{f}$ et $\mathrm{k}$ : secondes prémolaires inférieures droite (AP/89-2-69) et gauche (AP/89-2-65), g et $\mathrm{j}$ : canines inférieures droite (AP/89-2-64) et gauche (AP/89-2-70), h et $\mathrm{i}$ : incisives inférieures (AP/89-2-67 et AP/89-2-63). (clichés L. Chiotti) / Permanent teeth from P1 (scale: $2 \mathrm{~cm}$ ), lingual and labial views. a and e: upper right (AP/89-2-61) and left (AP/89-2-68) canines, b: upper right lateral incisor (AP/89-2-66). $c$ and $d$ : upper right (AP/89-2-62) and left (AP/89-2-71) central incisors, f and $k$ : lower right (AP/89-2-69) and left (AP/89-2-65) second premolars, $g$ and $j$ : lower right (AP/89-2-64) and left (AP/89-2-70) canines, $h$ and $i:$ lower incisors (AP/892-67 and AP/89-2-63). (images: L. Chiotti).

Tableau 3 Échantillon de comparaison pour les mesures des dents permanentes et déciduales / Comparative sample for permanent and deciduous tooth measurements.

\begin{tabular}{|llll|}
\hline Dents permanentes & Références & Dents déciduales & Références \\
\hline Arene Candide I & {$[26]$} & Dolní Věstonice 36/3 et 27 & {$[14]$} \\
Dolní Věstonice 3,13,14,15 & {$[14]$} & Lagar Velho 1 & {$[27]$} \\
Lagar Velho 1 & {$[27]$} & Paglicci 15 & {$[28]$} \\
Paglicci 15 & {$[28]$} & Pavlov 06/2, 12, 13, 14, 15 et 16 & {$[14]$} \\
Pavlov $1,3,22$ & {$[14]$} & & \\
Předmostí $1,2,3,4,5,7,9,10,14$ & {$[29]$} & & \\
\hline
\end{tabular}

2-63 serait à gauche, AP/89-2-67 à droite) mais ne permet pas d'être catégorique. De plus, ces dents rentrent mieux dans les alvéoles de la mandibule de P1 si elles sont considérées comme des incisives centrales (dans ce cas AP/892-63 serait à droite et AP/89-2-67 à gauche).

\section{Canines inférieures droite (AP/89-2-64) et gauche (AP/89-2-70)}

AP/89-2-64 est complète, avec une racine unique non altérée à l'apex fermé (dimension maximale : 24,9 mm). Cette dent s'articule bien avec la mandibule de P1. AP/89-2-70 était complète (dimension maximale : $24,0 \mathrm{~mm}$ ) mais sa racine a été prélevée pour datation [2].

Les deux dents présentent les mêmes caractéristiques morphologiques. L'usure du bord de la couronne est oblique dans le sens mésio-distal. Elle est assez faible et limitée à la pointe (stade 3). L'usure interproximale est marquée. Sur la face linguale en partie masquée par du sédiment et du consolidant on devine que les reliefs sont peu marqués. Sur la face vestibulaire, à un millimètre du collet, un petit dépôt de tarte est visible.

\section{Secondes prémolaires inférieures droite (AP/89-2-69) et gauche (AP/89-2-65)}

AP/89-2-69 est complète (dimension maximale : 24,4 mm) mais l'extrémité de la racine unique est fissurée. AP/89-2-65 est complète (dimension maximale : $23,6 \mathrm{~mm}$ ) mais une cassure transversale récente a séparé la dent en trois parties.

La couronne de AP/89-2-69 est de forme quadrangulaire avec deux cuspides principales, une vestibulaire et une mésiolinguale (la plus usée). Par rapport à cette cuspide, on note la présence de deux autres petites cuspides accessoires, 


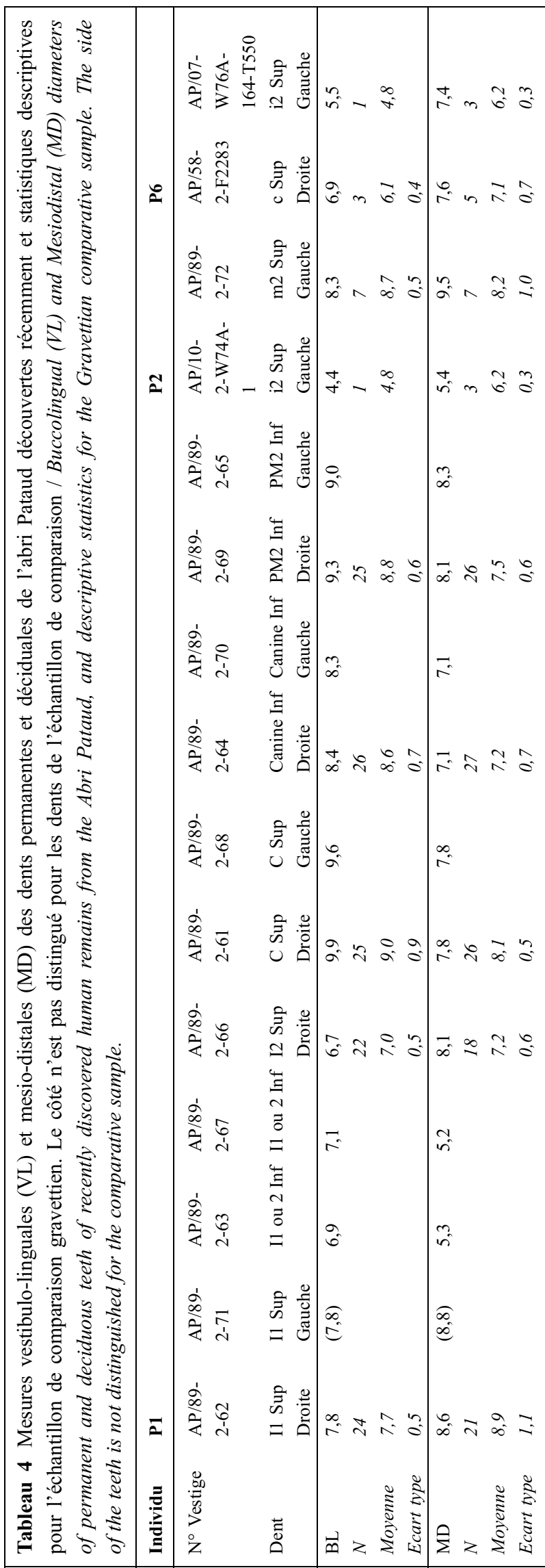

l'une en position mésiale, l'autre distolinguale. AP/89-2-65 est similaire en tout point à $\mathrm{AP} / 89-2-69$ si ce n'est l'absence de la petite cuspide accessoire en position mésiale.

L'usure de la couronne est faible à droite (stade 3), très faible à gauche (stade 2). Des facettes d'usure sont présentes sur les faces mésiale et distale des deux dents. On observe une ligne de tarte sur leurs faces vestibulaire et linguale.

\section{Vertèbres et côtes}

Les restes de vertèbres et de côtes attribués à P1 sont illustrés par les figures $3 a$ à 31 , et $4 a$ et $4 b$. Les mesures pour les vertèbres sont présentées dans le tableau 5 .

\section{Atlas (AP/89-2-309)}

Fragment d'atlas en 4 morceaux. Sont préservés la masse latérale gauche (dimension maximale : 24,6 mm), la moitié de l'arc postérieur gauche $(18,4 \mathrm{~mm})$, la masse latérale droite sub-complète $(24,4 \mathrm{~mm})$, et un fragment d'arc antérieur avec le tubercule antérieur et la surface articulaire pour la dent de l'axis $(18,3 \mathrm{~mm})$. La partie antérieure et latérale de la masse latérale gauche est très abimée. Les processus transverses sont cassés des deux côtés. Les surfaces articulaires inférieures sont incomplètes et leurs bords sont érodés.

La surface articulaire pour la dent de l'axis est ovoïde et concave. Les surfaces articulaires supérieures sont larges et concaves, avec deux facettes, non séparées. Les surfaces articulaires inférieures sont planes. Les tubercules qui servent à l'insertion du ligament transverse sont bien individualisés, à la surface lisse. Cet os s'articule bien avec l'axis de P1 (AP/63-1/2-1397B) et semble pouvoir s'articuler avec l'occipital de $\mathrm{P} 1$, mais le remontage reste difficile du fait de la fragmentation importante de l'atlas.

Autres vertèbres (AP/89-2-37967; AP/89-2-37974, 37975, 37976, 37977, 37978, 37979 et 37980 ; AP/89-2-288, 310 et 313)

AP/89-2-37979 est un fragment de pédicule de vertèbre cervicale 5 ou 6 (rang précisé grâce à la morphologie et par exclusion) (dimension maximale : 15,8 $\mathrm{mm}$ ).

$\mathrm{AP} / 89-2-288$ est un fragment de lame de vertèbre thoracique, détruit pour datation (dimension maximale : $38,9 \mathrm{~mm})$.

AP/89-2-37967 est un fragment de processus transverse droit de vertèbre thoracique avec la surface articulaire en partie préservée (dimension maximale : $8,3 \mathrm{~mm}$ ).

$\mathrm{AP} / 89-2-37974$ est fragment de vertèbre thoracique haute, avec la surface articulaire postérieure supérieure gauche, le pédicule gauche et une portion de corps sur laquelle est visible la surface articulaire costale gauche et une partie du plateau inférieur (dimension maximale : 25,2 mm). 


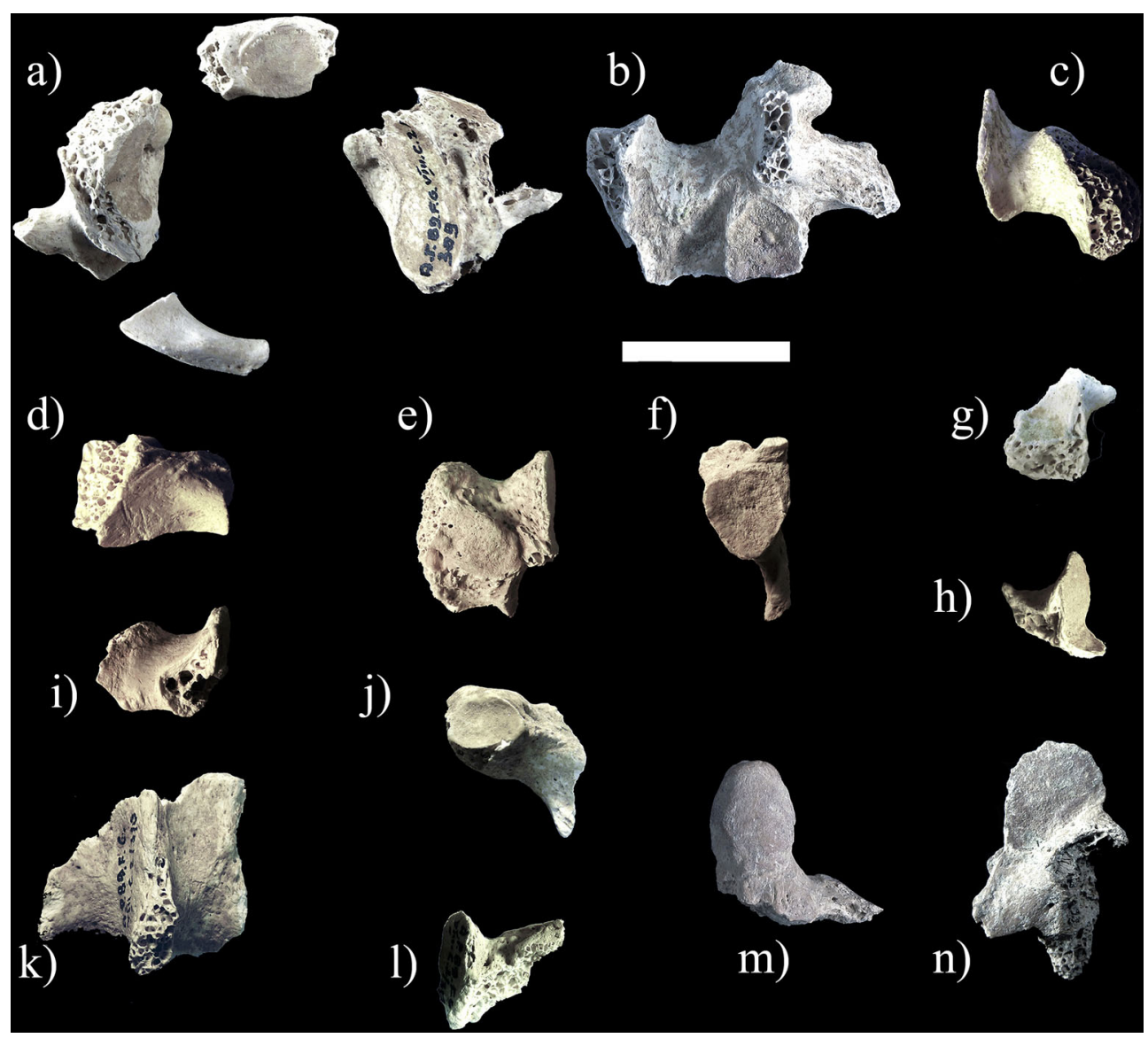

Fig. 3 Vertèbres adultes (échelle : $2 \mathrm{~cm}$ ). a : atlas (AP/89-2-309), vue supérieure, b : fragment de lame de vertèbre thoracique (AP/892-288), c : fragment de vertèbre thoracique haute (AP/89-2-37974), d : fragment de lame (AP/89-2-37975), e : Fragment de onzième vertèbre thoracique (AP/89-2-37977), f : fragment de processus postérieur inferieur droit d'une vertèbre thoracique (AP/89-2-37978), g : fragment de pédicule de vertèbre cervicale (AP/89-2-37979), h : processus postérieur supérieur droit de vertèbre thoracique (AP/89-2-37980), $\mathrm{i}$ : processus postérieur supérieur gauche de vertèbre thoracique (AP/89-2-37976), $\mathrm{j}$ : fragment de processus transverse (AP/89-2-37967), $\mathrm{k}$ : fragment de vertèbre thoracique basse (AP/89-2-310), 1 : fragment de processus postérieur supérieur droit d'une vertèbre thoracique (AP/89-2-313). $\mathrm{m}$ : fragment d'axis (AP/58-2-37966), $\mathrm{n}$ : fragment de vertèbre thoracique (AP/58-2-37958) (clichés S. Villotte, D. HenryGambier) / Adult vertebrae (scale: $2 \mathrm{~cm}$ ). a: atlas (AP/89-2-309), superior view, b: fragment of a lamina of a thoracic vertebra (AP/892-288), c: fragment of a upper thoracic vertebra (AP/89-2-37974), d: fragment of lamina (AP/89-2-37975), e: fragment of the 11th thoracic vertebra (AP/89-2-37977), $f$ : fragment of the right superior articular process of a thoracic vertebra (AP/89-2-37978), g: fragment of pedicle of a cervical vertebra (AP/89-2-37979), h: right superior articular process of a thoracic vertebra (AP/89-2-37980), $i$ : left superior articular process of a thoracic vertebra (AP/89-2-37976), $j$ : fragment of a transverse process (AP/89-2-37967), $k$ : fragment of a lower thoracic vertebra (AP/89-2-310), l: fragment of right superior articular process of thoracic vertebra (AP/89-2-313), m: fragment of axis (AP/582-37966), $n$ : fragment of a thoracic vertebra (AP/58-2-37958) (Images: S. Villotte, and D. Henry-Gambier).

AP/89-2-37975 représente une très petite portion supérieure d'une lame de vertèbre cervicale ou thoracique, avec une légère irrégularité au niveau de l'insertion des ligaments jaunes (dimension maximale : 18,9 mm).

AP/89-2-37976 consiste en un processus postérieur supérieur gauche de vertèbre thoracique (dimension maximale : $16,5 \mathrm{~mm})$.

AP/89-2-37977 correspond à la surface postérieure supérieure gauche, au pédicule gauche et à un fragment de corps d'une vertèbre thoracique basse, probablement la onzième (dimension maximale : 22,0 $\mathrm{mm}$ ).

AP/89-2-37978 est un fragment de processus postérieur inférieur droit d'une vertèbre thoracique, avec la surface articulaire préservée (dimension maximale : $22,5 \mathrm{~mm}$ ).

AP/89-2-37980 est un fragment de processus postérieur supérieur droit de vertèbre thoracique moyenne ou basse (dimension maximale : 13,0 $\mathrm{mm}$ ). 


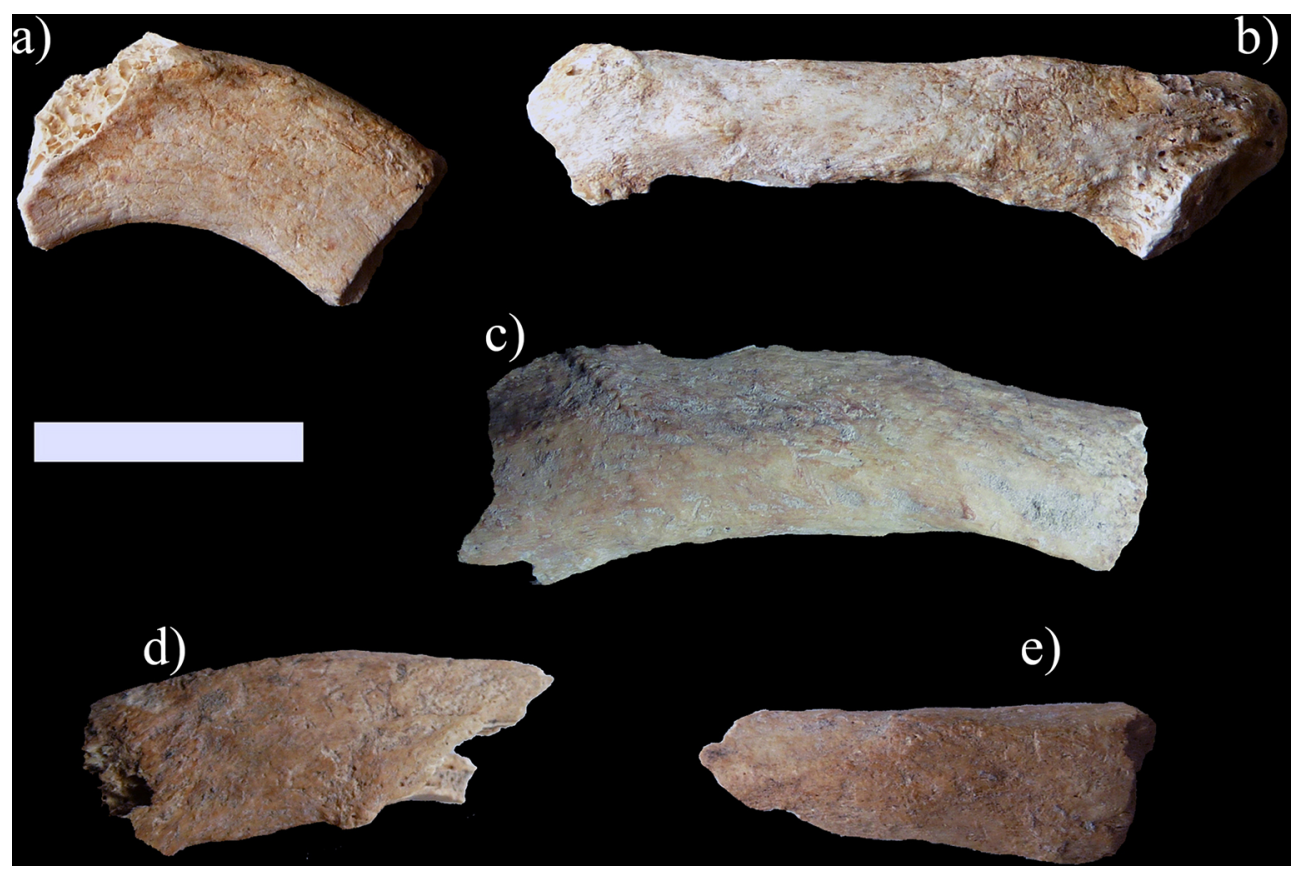

Fig. 4 Côtes adultes (échelle : $2 \mathrm{~cm}$ ). a : fragment de première côte droite (AP/89-2-37973), b : fragment de douzième côte gauche (AP/89-2-37299), c : fragment de côte droite (AP/58-2-37802), d : fragment de côte (AP/58-2-37803), e : fragment de côte (AP/582-37804) (clichés S. Villotte) / Adult ribs (scale: $2 \mathrm{~cm}$ ), a: fragment of a right first rib (AP/89-2-37973), b: fragment of a left 12th rib (AP/89-2-37299), c: fragment of a right rib (AP/58-2-37802), d: fragment of rib (AP/58-2-37803), e: fragment of rib (AP/58-2-37804) (Images: S. Villotte).

Tableau 5 Mesures des vertèbres. SA : Surface articulaire, FA : facette articulaire / Measurements for vertebrae. SA: Articular surface, FA: Articular facet.

\begin{tabular}{|c|c|c|c|c|c|c|c|c|c|}
\hline Individu & & & & 1 & & & P6 & PX17 & PX18 \\
\hline $\mathrm{N}^{\circ}$ vestige & $\begin{array}{l}\mathrm{AP} / 89- \\
2-309\end{array}$ & $\begin{array}{l}\text { AP/89- } \\
2-37974\end{array}$ & $\begin{array}{l}\text { AP/89- } \\
2-37978\end{array}$ & $\begin{array}{l}\mathrm{AP} / 89- \\
2-37980\end{array}$ & $\begin{array}{l}\mathrm{AP} / 89- \\
2-313\end{array}$ & $\begin{array}{l}\mathrm{AP} / 89- \\
2-310\end{array}$ & $\begin{array}{l}\mathrm{AP} / 58- \\
2-37956\end{array}$ & $\begin{array}{l}\mathrm{AP} / 58- \\
2-37966\end{array}$ & $\begin{array}{l}\mathrm{AP} / 58- \\
2-37958\end{array}$ \\
\hline Vertèbre & $\mathrm{C} 1$ & VT & VT & VT & VT & VT12 & $\mathrm{C} 1$ & $\mathrm{C} 2$ & VT \\
\hline Hauteur de la dent (M1a) & & & & & & & & 14,5 & \\
\hline $\begin{array}{l}\text { Diamètre max. antéro-post } \\
\text { érieur de la dent }\end{array}$ & & & & & & & & 11,0 & \\
\hline $\begin{array}{l}\text { Diamètre max. transverse } \\
\text { de la dent }\end{array}$ & & & & & & & & 10,6 & \\
\hline Hauteur de la SA de la dent & & & & & & & & 13,4 & \\
\hline Largeur de la SA de la dent & & & & & & & & 9,5 & \\
\hline Hauteur de la SA pour la dent & 9,7 & & & & & & 6,5 & & \\
\hline Largeur de la SA pour la dent & & & & & & & 10,5 & & \\
\hline Hauteur de la FA sup. droite & & & & 9,3 & 12,1 & & & & 12,8 \\
\hline Largeur de la FA sup. droite & 10,8 & & & & 9,6 & & & & 10,0 \\
\hline Hauteur de la FA sup. gauche & & & & & & & 20,8 & & \\
\hline Largeur de la FA sup. gauche & & $(11,9)$ & & & & & 8,6 & & \\
\hline Hauteur de la SA inf. droite & & & 11,2 & & & & & & \\
\hline Largeur de la FA inf. droite & & & 10,1 & & & 8,07 & & & \\
\hline Hauteur de la SA inf. gauche & & & & & & & 15,6 & & \\
\hline
\end{tabular}


$\mathrm{AP} / 89-2-310$ est un fragment de vertèbre thoracique basse, probablement la douzième, limité à la moitié inférieure de l'arc et aux deux surfaces articulaires inférieures incomplètes (dimension maximale : 30,4 mm).

$\mathrm{AP} / 89-2-313$ correspond à un fragment de processus postérieur supérieur droit (avec la surface articulaire préservée) d'une vertèbre thoracique, associé à une portion du pédicule et du corps (dimension maximale : 19,5 mm).

Les fragments ne présentent pas de caractères notables, et les surfaces articulaires n'ont pas d'atteintes. Une légère réaction périostée est présente au niveau du contour de la surface articulaire costale du fragment AP/89-2-37977.

\section{Côtes (AP/89-2-37973 et AP/89-2-299)}

AP/89-2-37973 est fragment de corps première côte droite, cassé après l'angle (dimension maximale : $32,6 \mathrm{~mm}$ ). La surface supérieure du fragment est irrégulière, la crête est bien développée et suivie antérieurement par un sillon large et peu profond pour l'insertion du muscle scalène moyen. AP/89-2-37299 correspond à l'extrémité vertébrale (érodée), au col et une portion du corps d'une côte gauche basse, probablement la douzième (dimension maximale : $55,8 \mathrm{~mm}$ ). La surface inférieure du corps est irrégulière et la surface articulaire présente des altérations sous la forme de porosités, de lacunes du cartilage calcifié, et de remodelages osseux.

\section{Os des membres supérieurs}

\section{Os du carpe (AP/63-2-37960 et AP/63-2-37970)}

AP/63-2-37960 correspond à l'extrémité latérale du tubercule du scaphoïde droit (dimension maximale : $15,5 \mathrm{~mm}$; fig. 5a). L'os est de petite dimension, symétrique du scaphoïde gauche de P1. AP/63-2-37970 est un trapézoïde droit sub-complet dont l'extrémité palmaire est cassée (dimension maximale : $18,8 \mathrm{~mm}$; fig. $5 \mathrm{~b}$ ). C'est un os de petite taille (Tableau 6), qui s'articule bien avec le capitatum droit de P1.

\section{Métacarpiens (AP/89-2-37971 ; AP/89-2-312 ; AP/89- 2-306)}

AP/89-2-37971 correspond à l'extrémité distale d'un métacarpien, peut-être le quatrième gauche (dimension maximale : 11,0 mm, fig. 5c). AP/89-2-312 correspond également à l'extrémité distale d'un métacarpien, peut être le cinquième droit (dimension maximale du fragment : 19,9 mm ; fig. 5d). AP/89-2-306 est un fragment de diaphyse de métacarpien, vraisemblablement le quatrième gauche, très gracile (dimension maximale : $29,4 \mathrm{~mm}$; fig. 5e). Les mesures sont présentées dans le tableau 7.
L'identification repose essentiellement sur la confrontation de ces trois fragments aux autres vestiges humains de l'abri Pataud, notamment les métacarpiens et métatarsiens de P1.

Phalanges de main (AP/89-2-302 ; AP/63-2-37962 ; AP/89-2-465)

AP/63-2-37962 correspond à l'extrémité proximale de la cinquième phalange moyenne gauche. Seuls la surface articulaire et son contour sont préservés (dimension maximale du fragment : $10,7 \mathrm{~mm}$; fig. $5 \mathrm{~g}$ ).

$\mathrm{AP} / 89-2-465$ est une cinquième phalange distale gauche sub-complète. L'extrémité proximale est érodée et l'extrémité distale est cassée (dimension maximale du fragment : $14.5 \mathrm{~mm}$; fig. 5h). Elle est très gracile.

$\mathrm{AP} / 89-2-302$ correspond à l'extrémité distale de la quatrième phalange proximale gauche (dimension maximale : $22,8 \mathrm{~mm}$; fig. $5 \mathrm{i})$.

Les mesures sont présentées dans le tableau 8 .

\section{Os des membres inférieurs}

\section{Métatarsien IV droit (?) (AP/63-2-37972)}

Il s'agit d'un fragment d'extrémité distale de métatarsien, avec la surface articulaire érodée (dimension maximale du fragment : $11,5 \mathrm{~mm}$; fig. $5 \mathrm{k}$ ). Il s'agit vraisemblablement du quatrième métatarsien droit.

\section{Autres vestiges d'adultes}

Ces ossements sont des vestiges qui n'ont pas été attribués à un des trois individus adultes (P1, P3 ou P5) dans HenryGambier et al. [6].

\section{Vestiges crâniens}

\section{Os zygomatique et maxillaire droits (AP/58-2-37954)}

L'os zygomatique est presque complet (Fig. 1b) ; il lui manque le processus temporal, ainsi que l'extrémité médiale et la partie postérieure du processus orbitaire. L'os maxillaire associé est représenté par son processus zygomatique. Dimension maximale du fragment : $57,2 \mathrm{~mm}$.

Les os ont une morphologie totalement mature. L'os zygomatique présente deux foramens zygomatico-faciaux et deux foramens zygomatico-orbitaux qui correspondent entre eux par deux canaux indépendants (Fig. 1b). Sous les foramens zygomatico-faciaux, de nombreux petits foramens sont visibles. Un seul foramen, avec un large diamètre (environ $2 \mathrm{~mm}$ ), est observable sur la face temporale. 


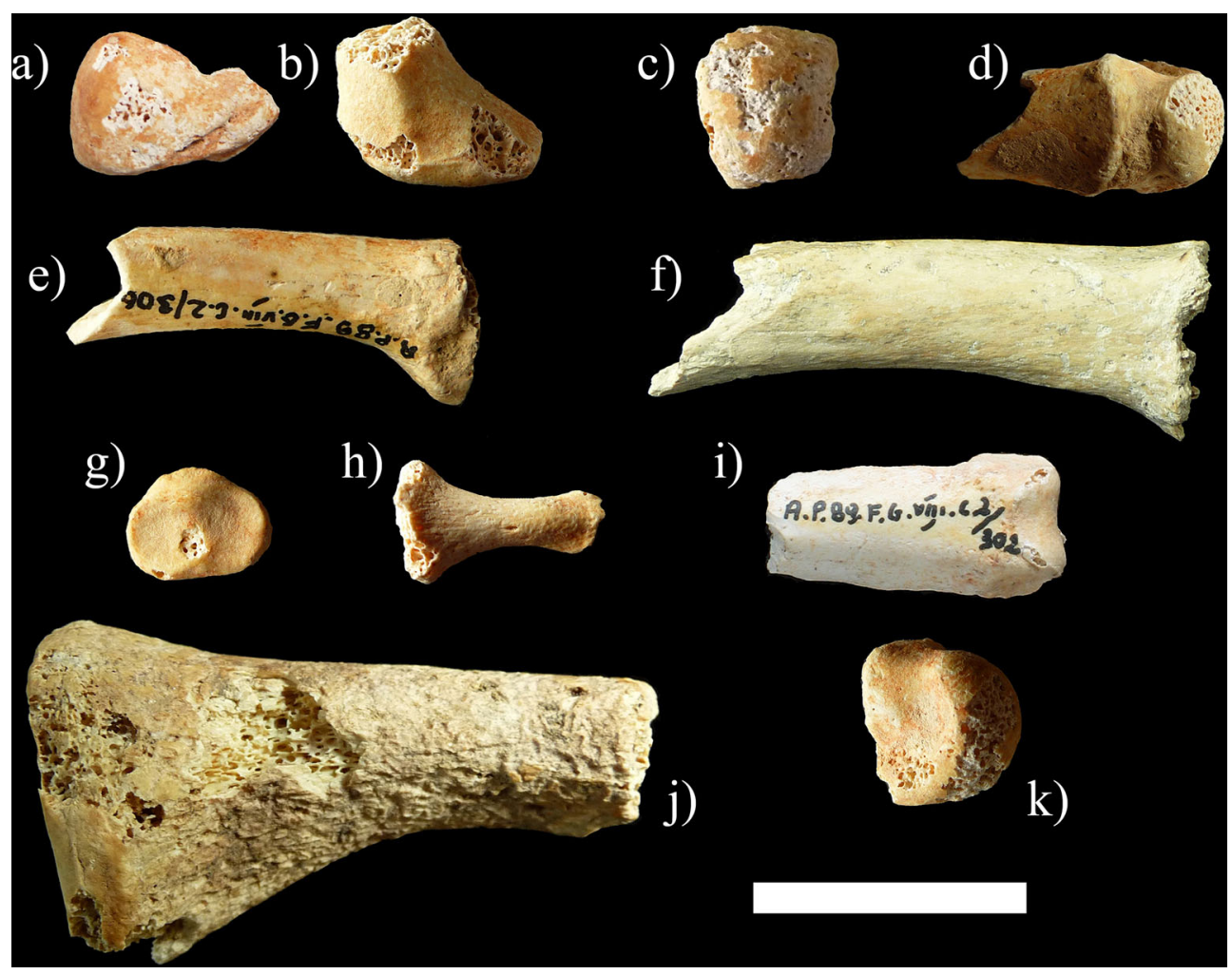

Fig. 5 Ossements des mains et des pieds (échelle : $2 \mathrm{~cm}$ ). a : tubercule du scaphoïde droit (AP/63-2-37960), b : trapézoïde droit (AP/632-37970), c : tête de métacarpien (AP/89-2-37971), d : extrémité distale de métacarpien (AP/89-2-312), e : fragment de diaphyse de métacarpien (AP/89-2-306), f : métacarpien III droit (AP/58-2-37964), g : extrémité proximale de phalange moyenne gauche de main V (AP/63-2-37962), h : phalange distale gauche de main V (AP/89-2-465), i : l'extrémité distale de quatrième phalange proximale gauche de main (AP/89-2-302), j : métatarsien I gauche (AP/--37801), k : extrémité distale de métatarsien IV droit (AP/63-2-37972) (clichés S. Villotte) / Hand and foot bones (scale: $2 \mathrm{~cm}$ ). a: Right scaphoid tubercle (AP/63-2-37960), b: Right trapezoid (AP/63-2-37970), c): metacarpal head (AP/89-2-37971), $d$ : distal part of a metacarpal (AP/89-2-312), e: fragment of shaft of a metacarpal (AP/89-2-306), f: right third metacarpal (AP/58-2-37964), g: proximal part of left fifth middle phalanx of the hand (AP/63-2-37962), h: left fifth distal phalanx of the hand (AP/89-2-465), i: Distal part of left fourth proximal phalanx of the hand (AP/89-2-302), j: left first metatarsal (AP/-37801), $k$ : distal part of right fourth metatarsal (AP/63-2-37972) (Images: S. Villotte)

Tableau 6 Mesures du trapézoïde droit AP/63-2-37970 attribué à $\mathrm{P} 1$ et comparaison avec celles de l'os gauche de P3 conservé au Musée de l'Homme / Measurements for the right trapezoid AP/63-2-37970 assigned to P1 and comparison with those of the left trapezoid of P3 curated in the Musée de l'Homme.

\begin{tabular}{|lll|}
\hline Individu & P1 & P3 \\
\hline $\mathrm{N}^{\circ}$ vestige & AP/63-2-37970 & \\
Côté & Droit & Gauche \\
\hline Longueur maximale (M1) & 16,1 & 16,0 \\
Largeur maximale (M2) & 11,0 & 9,9 \\
Largeur de la surface & 10,1 & 6,7 \\
articulaire pour le capitatum & & \\
(M8) & & \\
\hline
\end{tabular}

Le bord orbitaire du zygomatique est proéminent sans être particulièrement épais. Le processus frontal présente un tubercule marginal peu prononcé ("weak" dans Hauser et De Stefano [31]) (Fig. 1b). Le bord inférieur de la face latérale est rugueux au niveau de l'insertion du muscle masséter. L'os maxillaire et la suture zygomaxillaire ne présentent pas de morphologie particulière.

\section{Os temporal gauche (AP/58-2-37963)}

Il s'agit de la partie antéro-inférieure de l'os (dimension maximale : 50,0 mm ; Fig. 1c). La partie pétreuse, une grande part de la partie squameuse, ainsi que les processus mastoïde et styloïde sont manquants. Le bord sphénoïdal est préservé.

Le fragment présente plusieurs traits notables. Le principal est un foramen au niveau de la surface articulaire de la fosse mandibulaire. Ce caractère semble rare et peu décrit 
Tableau 7 Mesures des métacarpiens / Measurements of metacarpal bones.

\begin{tabular}{|llll|}
\hline Individu & & P1 & PX31 \\
\hline$N^{\circ}$ vestige & AP/89-2-306 & AP/89-2-312 & AP/58-2-37964 \\
Os & MTC IV gauche ? & MTC V droit ? & MTC III droit \\
\hline Hauteur à mi diaphyse (M7) & 7,1 & & 9,9 \\
Largeur à mi diaphyse (M6) & 5,9 & & 8 \\
Hauteur minimum du corps & & & 9,7 \\
Largeur minimum du corps & & 13,5 & 7,8 \\
Hauteur distale (M11) & & 10,8 & \\
Largeur distale (M9) & 10,5 & \\
Largeur de la surface articulaire de la tête & & \\
\hline
\end{tabular}

Tableau 8 Mesures des phalanges des mains / Measurements of hand phalanges.

\begin{tabular}{|llll|}
\hline Individu & & \multicolumn{1}{c|}{ P1 } \\
\hline $\mathrm{N}^{\circ}$ vestige & AP/89-2-302 & AP/63-2-37962 & AP/89-2-465 \\
Os & $\begin{array}{l}\text { Phalange proximale gauche } \\
\text { de main IV }\end{array}$ & $\begin{array}{l}\text { Phalange moyenne gauche } \\
\text { de main V }\end{array}$ & gauche de main V \\
\hline Hauteur à mi diaphyse (M7) & & & 3,0 \\
Largeur à mi diaphyse (M6) & & & 3,4 \\
Hauteur distale (M11) & 7,9 & & 3,2 \\
Largeur distale (M9 & 11,1 & & 4,4 \\
Largeur articulaire distale & 8,6 & 8,3 & 5,3 \\
Hauteur proximale (M10) & & 10,7 & 8,5 \\
Largeur proximale (M8) & & 7,5 & 10,3 \\
Hauteur articulaire proximale & & & \\
Largeur articulaire proximale & & & \\
\hline
\end{tabular}

[32]. La partie squameuse présente deux foramens sur sa face externe et quatre sur sa face interne. Les deux foramens de la face externe sont situés $3 \mathrm{~mm}$ environ au-dessus de la fosse mandibulaire. En interne, un premier foramen est recouvert par la lame osseuse irrégulière. Un deuxième est associé à une dépression ovalaire de la surface endocrânienne. Deux autres petit foramens sont observables, l'un deux étant également associé à une petite dépression ovalaire. Seul le deuxième semble déboucher quelque part : il correspond avec le plus gros des deux foramens de la face externe. La surface endocrânienne présente enfin une petite production osseuse irrégulière d'environ $2 \mathrm{~mm}$ de diamètre. L'origine de ces modifications est inconnue.

La morphologie générale des fragments AP/58-2-37954 et $\mathrm{AP} / 58-2-37963$, l'aspect très similaire de leur surface, et la présence de foramens surnuméraires dans les deux cas plaident en faveur de l'appartenance à un même individu.

\section{Vertèbres et côtes}

Ces fragments sont illustrés par les figures 3 et 4, les mesures vertébrales sont présentées dans le tableau 5 .

\section{Axis (AP/58-2-37966)}

Fragment limité à la dent de l'axis et au bord médial de la surface articulaire supérieure gauche (dimension maximale : 22,3 mm ; Fig. 3m).

La surface articulaire de la dent est légèrement prolongée supérieurement et à son bord inféro-latéral gauche. Ce léger débordement pourrait correspondre un à stade très peu avancé d'ostéophytose marginale. Le sillon postérieur (concavité) de la dent, pour le ligament transverse de l'atlas, est très marqué.

\section{Vertèbre thoracique (AP/58-2-37958)}

Le processus articulaire supérieur droit, un fragment du pédicule et de la lame, et une petite partie de la surface articulaire postéro-inférieure droite sont préservés (dimension maximale : $27,4 \mathrm{~mm}$; fig. $3 \mathrm{n}$ ).

Il s'agit vraisemblablement d'une vertèbre thoracique haute, qui a pour seule particularité de présenter une petite production osseuse au niveau de l'insertion des ligaments jaunes. 


\section{Côtes (AP/58-2-37802, 37803 et 37804)}

AP/58-2-37802 est une section de corps d'une côte droite avec la partie distale de l'angle conservée (dimension maximale : 48,4 mm ; fig. 4c). AP/58-2-37803 correspond à un fragment de corps d'une dimension maximale de $35,8 \mathrm{~mm}$ (Fig. 4d). AP/58-2-37804 est un fragment de côte dont seule la surface externe est préservée, d'une longueur maximale de 33,0 mm (Fig. 4e). Ces os ne présentent pas de caractéristiques morphologiques notables.

\section{Os des membres supérieurs}

\section{Scapula gauche (AP/58-2-592)}

Cet os est endommagé (dimension maximale : 107,4 mm). Sont présents : une partie du corps, la cavité glénoïde (dont la marge inférieure est érodée), l'épine (son bord postérieur étant manquant médialement), un fragment de l'acromion (cassé au niveau de la cavité glénoïde), une partie du bord latéral (sur $60 \mathrm{~mm}$ environ), et la base du processus coracoïde (Fig. 6).

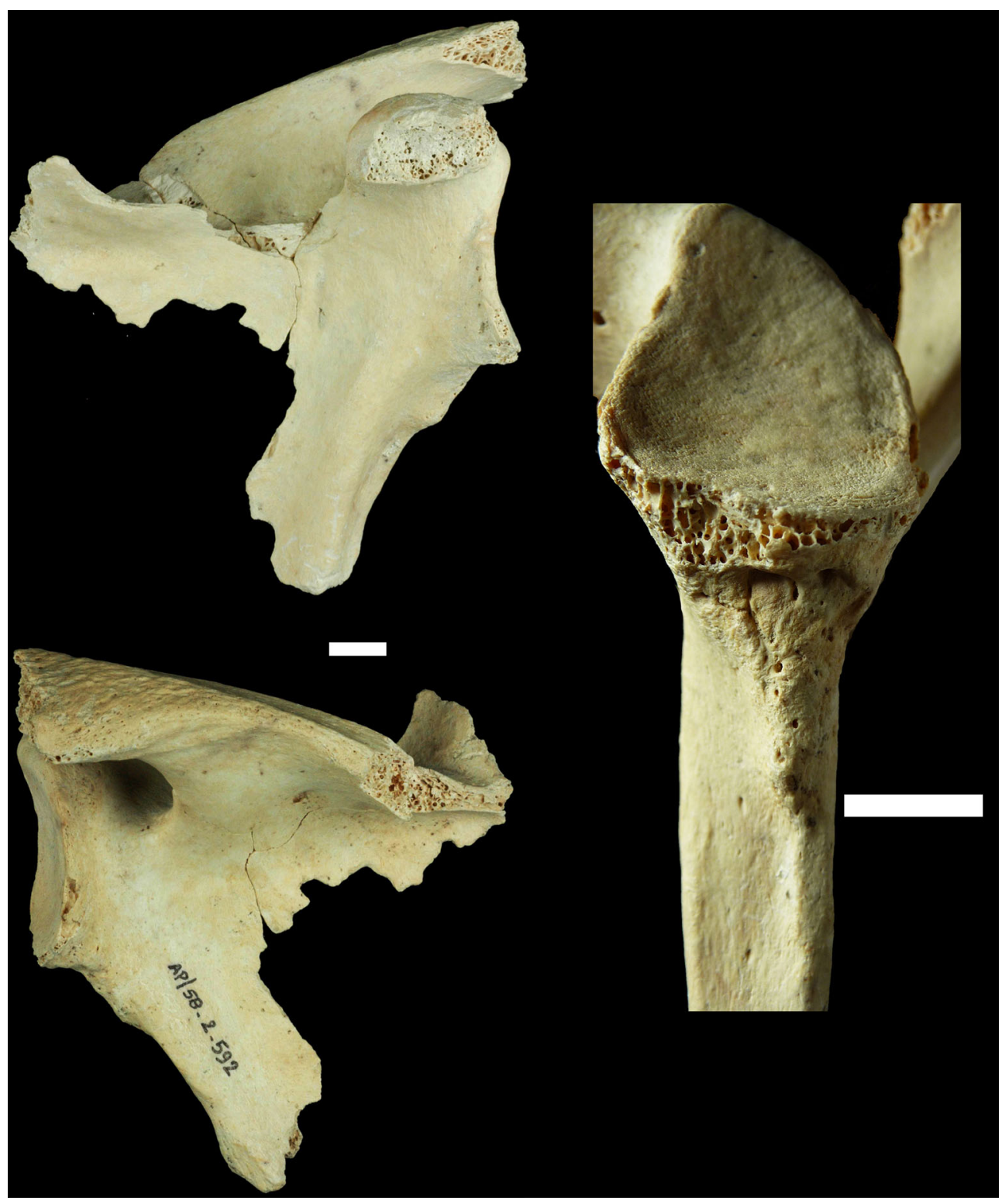

Fig. 6 Scapula gauche (AP/58-2-592) (échelles : $1 \mathrm{~cm})$. Vues antérieure, postérieure et vue rapprochée du bord latéral et de la cavité glénoïde (clichés S. Villotte) / Left scapula (AP/58-2-592) (scales: $1 \mathrm{~cm}$ ). Anterior, posterior views, and close up view of the lateral border and the glenoid cavity (Images: S. Villotte). 
L'os apparait assez robuste, avec une épine volumineuse (Tableau 9 et Fig. 6). La marge postérieure de celle-ci est manquante médialement mais une projection vers l'arrière dans cette région peut être supposée d'après la forme du bord. Cette morphologie a déjà été observée pour d'autres sujets gravettiens [33], et pour la scapula du sujet P3 de l'abri Pataud [8]. La partie latérale du bord postérieur de l'épine présente inférieurement un sillon très marqué (4 mm de largeur pour une longueur de $30 \mathrm{~mm}$ environ). Cette zone pourrait correspondre à l'insertion du muscle deltoïde. La surface postérieure, sous l'épine, présente un foramen de grande dimension et un sillon assez marqué qui court vers le bas et le côté médial. Au-dessus de l'épine, un autre foramen, beaucoup plus petit, est visible.

La cavité glénoïde, piriforme, à concavité antéro-post érieure, présente une échancrure antérieure bien marquée. Elle est d'assez grandes dimensions (Fig. 7). La surface articulaire est régulière et lisse mais un léger débordement marginal ostéophytique est observable antérieurement et postérieurement (Fig. 6). La marge postérieure présente également dans sa partie inférieure des altérations ostéolytiques régulières qui évoquent des géodes. Le tubercule infraglenoïde est long et saillant, avec un surcreusement dans sa partie antérieure, dans lequel se situe un foramen. Le bord latéral présente un sillon ventral large (environ $9 \mathrm{~mm}$ ) et un renfort ventral important. Cette morphologie de bord axillaire (sillon ventral) est assez rarement observée pour le Paléolithique supérieur (par ex. : 17,2\% dans Trinkaus et al. [25]). La morphologie de cet os le distingue très nettement de la scapula droite de P3. Il apparaît beaucoup plus robuste et de dimensions légèrement plus grandes, avec notamment une cavité glénoïde plus développée dans le plan coronal, un sillon sur le bord latéral de la scapula plus marqué et ventral, un tubercule infra glénoïdien plus saillant, et un processus coracoïde beaucoup plus épais (Tableau 9).
Les modifications articulaires et les dimensions semblent indiquer l'appartenance à un adulte assez robuste et plutôt âgé, ce qui exclut P1. Il semble donc justifié d'associer cet os à P5. Malheureusement, comme l'extrémité proximale de l'humérus gauche de P5 est manquante, la congruence articulaire entre les deux os ne peut être testée.

\section{Clavicule gauche (AP/58-2-F1026A)}

Il s'agit de la moitié médiale de la diaphyse (Fig. 8a). La partie conservée s'étend de l'impression pour le ligament costo-claviculaire (sub-complète) au milieu de la diaphyse (dimension maximale : 70,3 mm).

L'os est robuste, à section diaphysaire ovoïde développée antéro-postérieurement (Fig. 9), et avec une surface inférieure plate à légèrement concave. La partie médiale de la face antérieure est plate et légèrement irrégulière pour l'insertion du muscle grand pectoral. La marge inférieure de cette zone est délimitée par une crête. L'impression pour le ligament costo-claviculaire est légèrement irrégulière.

La morphologie de cet os le distingue nettement de la clavicule droite de P1, notamment par ses dimensions (Tableau 10) et par la présence d'une crête marquée à la jonction des faces inférieure et antérieure. P3 ayant déjà ces deux clavicules, il est probable que cet os appartienne à P5 [6].

\section{Clavicule droite (AP/58-2-F1026B)}

Tiers latéral de la diaphyse (Fig. 8b) dont l'extrémité acromiale est manquante (dimension maximale : $50,5 \mathrm{~mm}$ ). L'os apparait assez robuste (Tableau 10). La surface d'insertion pour le muscle deltoïde est bien délimitée, concave et très légèrement rugueuse dans la partie latérale, rugueuse et saillante dans sa partie médiale. Le tubercule conoïde forme un

Tableau 9 Mesures de la scapula gauche AP/58-2-592 et comparaison avec celles de la scapula droite de P3 conservée au Musée de l'Homme. La position pour les épaisseurs du bord axillaire et de l'épine de AP/58-2-592 est estimée / Measurements for the left scapula AP/58-2-592 and comparison with P3 right scapula curated in the Musée de l'Homme. The location to measure thickness of the axillary border and thickness of the spine of AP/58-2-592 is estimated.

\begin{tabular}{|c|c|c|}
\hline Individu & PX 13 & P3 \\
\hline $\mathrm{N}^{\circ}$ vestige & $\mathrm{AP} / 58-2-592$ & \\
\hline Côté & Gauche & Droit \\
\hline Epaisseur du bord axillaire & 12,4 & 11,4 \\
\hline Epaisseur de l'épine & 13,4 & 12,1 \\
\hline Largeur de l'acromion à la base & 27,4 & 30,2 \\
\hline Hauteur maximale de la cavité glénoïde (M12) & 41,5 & 38,5 \\
\hline Largeur maximale de la cavité glénoïde (M13) & 27,0 & 27,0 \\
\hline Hauteur de la surface articulaire de la cavité glénoïde & 37,2 & 37,7 \\
\hline Largeur de la surface articulaire de la cavité glénoïde & 24,5 & \\
\hline Morphologie du bord medial & Sillon ventral & Deux sillons \\
\hline
\end{tabular}




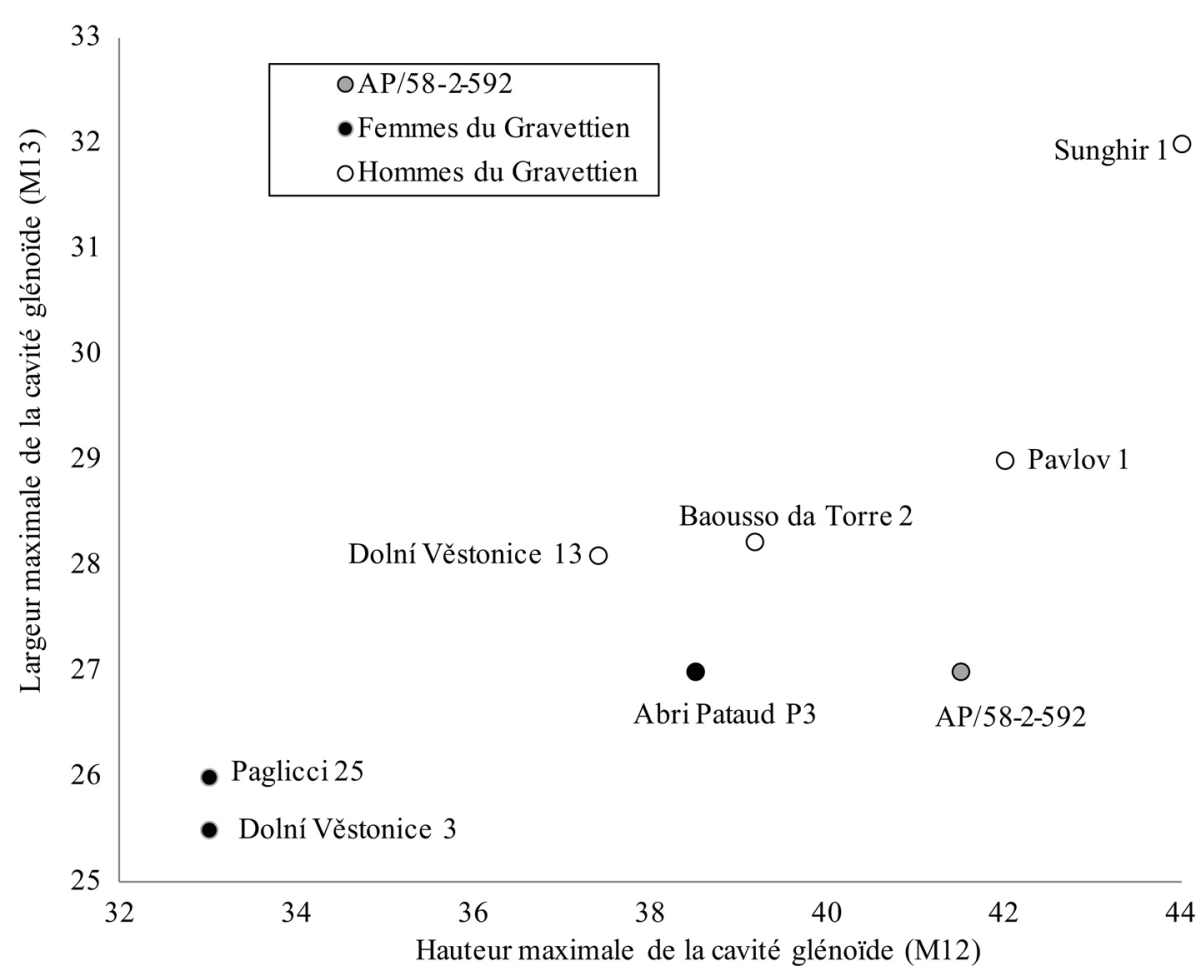

Fig. 7 Cavité glénoïde. Largeur maximale par rapport à la hauteur maximale pour l'abri Pataud et les autres fossiles du Gravettien (mesures du côté gauche pour tous les individus sauf abri Pataud P3, Dolní Věstonice 3, et Sunghir 1) / Glenoid cavity. Maximum breadth versus maximum height for Abri Pataud human remains and other Gravettian fossils (measures for the left side for all individuals but Abri Pataud P3, Dolní Věstonice 3, and Sunghir 1).

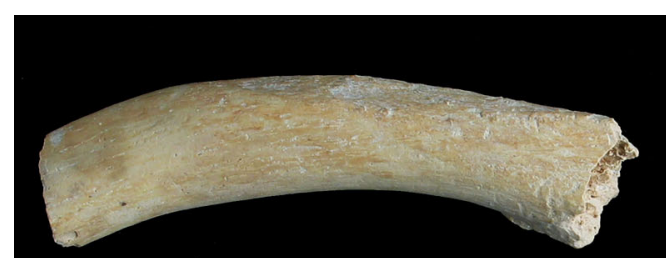

a)

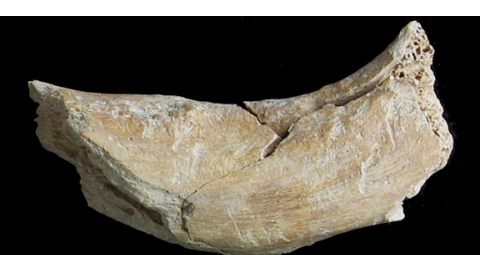

b)

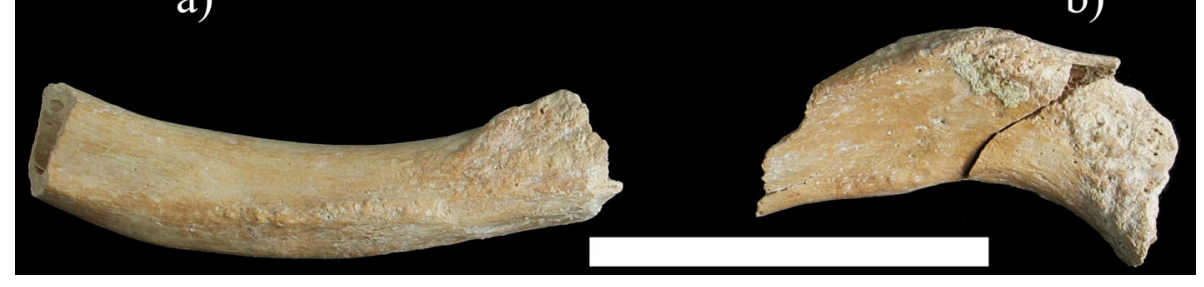

Fig. 8 Clavicules gauche (a : F1026A) et droite (b : F1026B) (échelle : $5 \mathrm{~cm}$ ). Vues supérieure et inférieure (clichés S. Villotte) / Left (a: F1026A) et right (b: F1026B) clavicles (scale: $5 \mathrm{~cm}$ ). Superior and inferior views (Images: S. Villotte).

relief bien distingué et irrégulier. La zone d'attachement du ligament trapézoïde est également rugueuse.

Comme $\mathrm{P} 1$ et $\mathrm{P} 3$ ont déjà une clavicule droite, il est probable que cet os appartienne à $\mathrm{P} 5$ [6]. La morphologie générale, similaire à $\mathrm{AP} / 58-2-\mathrm{F} 1026 \mathrm{~A}$, ne contredit pas cette hypothèse.

\section{Métacarpien III droit (AP/58-2-37964)}

Fragment limité à la diaphyse (dimension maximale : 40,0 mm ; Fig. 5f ; Tableau 7).

La surface dorsale est légèrement irrégulière au niveau de l'attachement des muscles interosseux dorsaux. L'aspect de 


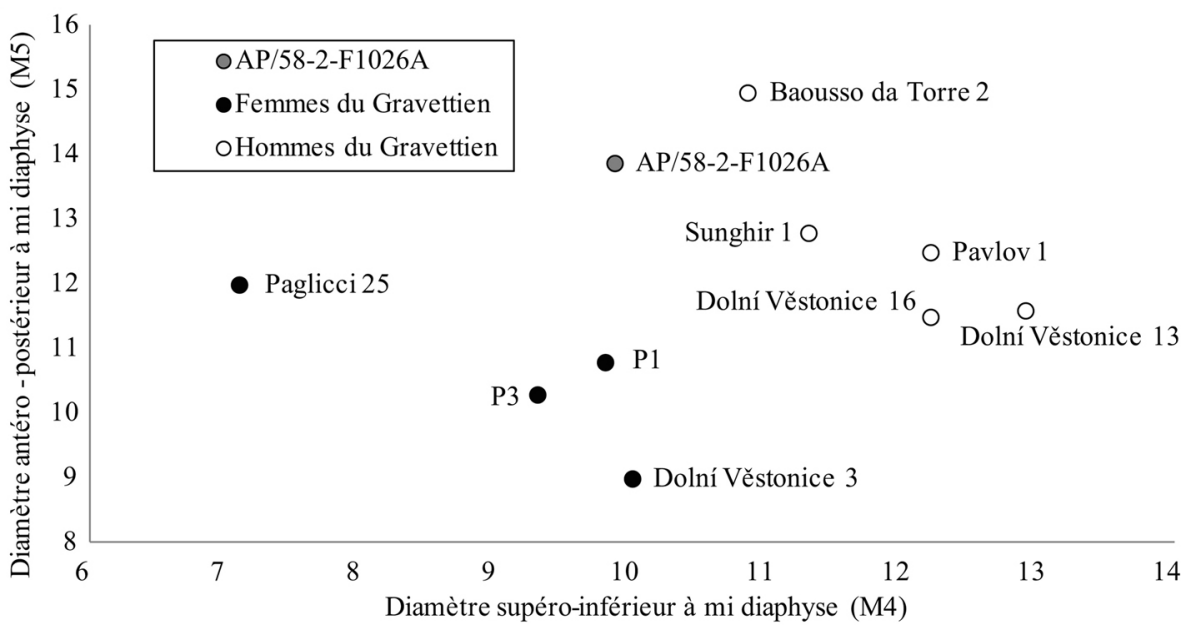

Fig. 9 Clavicule à mi diaphyse. Diamètre antéro-postérieur par rapport au diamètre supéro-inférieur pour l'Abri Pataud et les autres fossiles du Gravettien (mesures du côté gauche pour tous les individus sauf abri Pataud P1) / Clavicle at midshaft. Anterposterior versus superoinferior diameters for Abri Pataud human remains and other Gravettian fossils (measures for the left side for all individuals but Abri Pataud P1).

Tableau 10 Dimensions de clavicules adultes AP/58-2-F1026A et B et immature AP/58-2-37959, et comparaison avec celles de P1 et P3 conservées au Musée de l'Homme / Measurements for adult clavicles AP/58-2-F1026A and B, and immature clavicle AP/582-37959, and comparison with those of P1 and P3 curated in the Musée de l'Homme.

\begin{tabular}{|c|c|c|c|c|c|c|}
\hline Individu & PX 14 & PX15 & P1 & & P3 & P6 \\
\hline $\mathrm{N}^{\circ}$ vestige & $\begin{array}{l}\mathrm{AP} / 58- \\
2-\mathrm{F} 1026 \mathrm{~A}\end{array}$ & $\begin{array}{l}\mathrm{AP} / 58- \\
2-\mathrm{F} 1026 \mathrm{~B}\end{array}$ & & & & $\begin{array}{l}\text { AP/58- } \\
2-37959\end{array}$ \\
\hline Côté & Gauche & Droit & Droit & Droit & Gauche & Gauche \\
\hline Diamètre max. à mi diaphyse & 14,2 & & 11,3 & 12,3 & 10,6 & 6,5 \\
\hline Diamètre min. à mi diaphyse & 9,6 & & 9,8 & 10,4 & 9,3 & 4,5 \\
\hline Diamètre supéro-inférieur à mi diaphyse (M4) & 9,9 & & 9,8 & 10,4 & 9,3 & 4,6 \\
\hline Diamètre antéro-postérieur à mi diaphyse (M5) & 13,9 & & 10,8 & 11,1 & 10,3 & 6,4 \\
\hline Circonférence à mi diaphyse (M6) & 39,5 & & 34,0 & 36,0 & 33,0 & 19,0 \\
\hline Diamètre supero-inférieur à la tubérosité conoïde & & 12,6 & 8,4 & 10,5 & 9,8 & \\
\hline Diamètre antéro-postérieur à la tubérosité conoïde & & 18,8 & 16,5 & 17,4 & 17,2 & \\
\hline
\end{tabular}

l'enthèse ne correspond pas avec ce qui est observé pour P1 et P3 au niveau de leurs métacarpiens. Il pourrait donc appartenir à P5.

\section{Os des membres inférieurs}

\section{Fémur droit (AP/58-2-1011)}

Fragment de diaphyse cassé dans sa partie proximale juste en dessous du niveau sous-trochantérien (dimension maximale : 89,3 mm ; Fig. 10).

En face postérieure, sont visibles médialement la partie distale de ligne pectinée et la moitié distale de la surface d'attachement du muscle grand fessier. Cette zone est concave et légèrement rugueuse. La ligne âpre est irrégu- lière, le pilastre est modestement développé, avec une concavité latérale. Dans la partie proximale du fragment, l'extrémité distale du renfort latéral est visible. Pour autant qu'il soit possible d'en juger sur ce fragment, ce renfort latéral semble très faiblement développé, alors qu'il est souvent très important chez les sujets gravettiens, p. ex. [25,34]. A la cassure proximale, le diamètre transverse est de $29.7 \mathrm{~mm}$ et le diamètre antéro-postérieur est de $23,0 \mathrm{~mm}$, ce qui permet d'estimer un diamètre transverse sous trochantérien (M9) compris entre 29,5 et $31,5 \mathrm{~mm}$ et un diamètre antéropostérieur sous trochantérien (M10) compris entre 23,0 et 25,0 mm. Ces dimensions, même estimées, restent très petites le Gravettien. AP/58-2-1011 est plus petit que tous les autres sujets pour M9 et que tous, sauf Dolní Věstonice 3 (un sujet féminin), pour M10 (Fig. 11). 


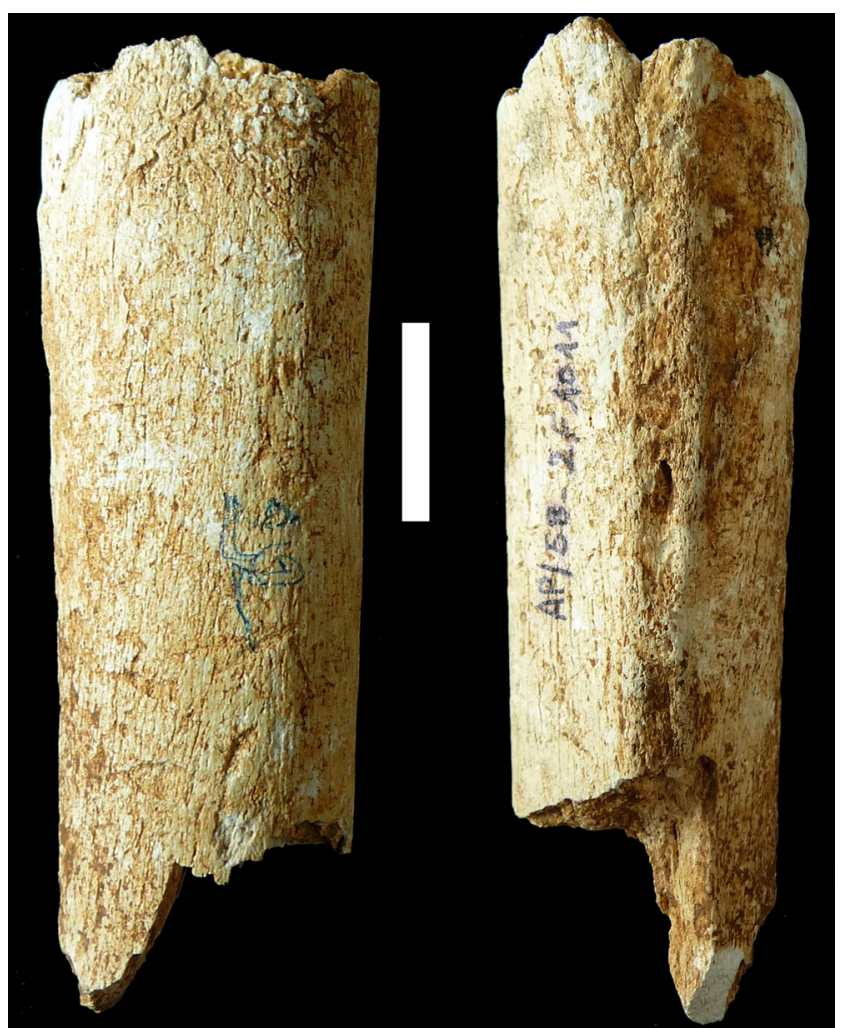

Fig. 10 Fémur droit (AP/58-2-1011) (échelle : $2 \mathrm{~cm})$. Vues antérieure et postérieure (cliché S. Villotte) / Right femur (AP/582-1011) (scale: $2 \mathrm{~cm}$ ). Anterior and posterior views (Images: S. Villotte).

\section{Métatarsien I gauche (AP/--37801)}

La base et les deux tiers proximaux de la diaphyse sont conservés (dimension maximale : 45,6 mm ; Fig. 5j). Les contours de la base sont légèrement érodés. L'os présente peu de caractéristiques morphologiques notables. Il est de petites dimensions (Tableau 11). La base est légèrement concave transversalement et la diaphyse est très aplatie supé ro-inférieurement.

\section{Vestiges immatures attribués à $\mathbf{P 2}$}

\section{Dents}

Les mesures sont présentées dans le tableau 4.

\section{Incisive latérale déciduale supérieure gauche (AP/10-2-W74A-1)}

Germe limité à la couronne dont la surface n'est pas altérée (dimension maximale : 8,0 mm ; Fig. 12a). On n'observe aucun caractère morphologique particulier, à part une dépression assez marquée au niveau distal de la surface linguale et un volume relativement important de la couronne (Tableau 4). Le degré de minéralisation dentaire (stade $\mathrm{Crc}$ ) correspond à un âge dentaire de 4,5 mois $+/-3$ mois [35].

\section{Seconde molaire déciduale supérieure gauche (AP/89-2-72)}

Germe limité à une partie de la couronne (dimension maximale : $8,9 \mathrm{~mm}$; Fig. 12b). La couronne, formée à un tiers environ $(4 \mathrm{~mm})$, comprend 4 cuspides bien individualisées. Le degré de minéralisation dentaire (stade $\mathrm{Coc}$ ) correspond à un âge dentaire de 4,5 mois $+/-3$ mois [35].

\section{Vertèbres}

\section{Vertèbre lombaire (AP/08-2-W76C-140)}

Deux hémi-arcs neuraux, soudés et cassées sur place, de vertèbre lombaire (1 à 4) (dimensions maximales des hémi-arcs droit et gauche : respectivement 18,5 et 17,6 mm). Présence des deux lames et des deux pédicules avec les surfaces métaphysaires pour le corps érodées mais en partie visible (Fig. 13c). Les surfaces articulaires postérieures supérieures et inférieures sont visibles mais érodées. Toute la partie médiane des lames est érodée.

Le corps n'était pas soudé et la soudure des hémi-arcs neuraux était partielle, indiquant un âge compris entre 6 mois et 2 ans [36].

\section{Os des membres supérieurs}

\section{Humérus droit et gauche (AP/89-2-293 et AP/58-2-37955)}

Les deux fragments correspondent à la moitié distale des diaphyses humérales immatures droite et gauche (dimensions maximales : respectivement 40,0 et $41,0 \mathrm{~mm}$; Fig. $14 \mathrm{c}$ ). La fosse olécranienne et la surface métaphysaire inférieure (en partie érodée) sont visibles à droite, absentes à gauche.

Les mesures sont présentées dans le tableau 12. L'os droit présente deux principaux foramens nourriciers sur sa face médiale. La fosse olécranienne est bien creusée, alors que la fosse coronoïde est très peu marquée. Un léger sillon est visible sur la face médiale de la diaphyse. La morphologie de ce sillon, qui évoque celle d'une enthèse immature [37], pourrait correspondre à la zone d'insertion de la marge médiale du muscle brachialis. A gauche un seul foramen est visible, et un sillon est également présent.

\section{Os des membres inférieurs}

\section{Pubis gauche (?) (AP/63-2-37961)}

Ce fragment pourrait correspondre à la branche supérieure et la surface acétabulaire (convexe et avec un aspect 


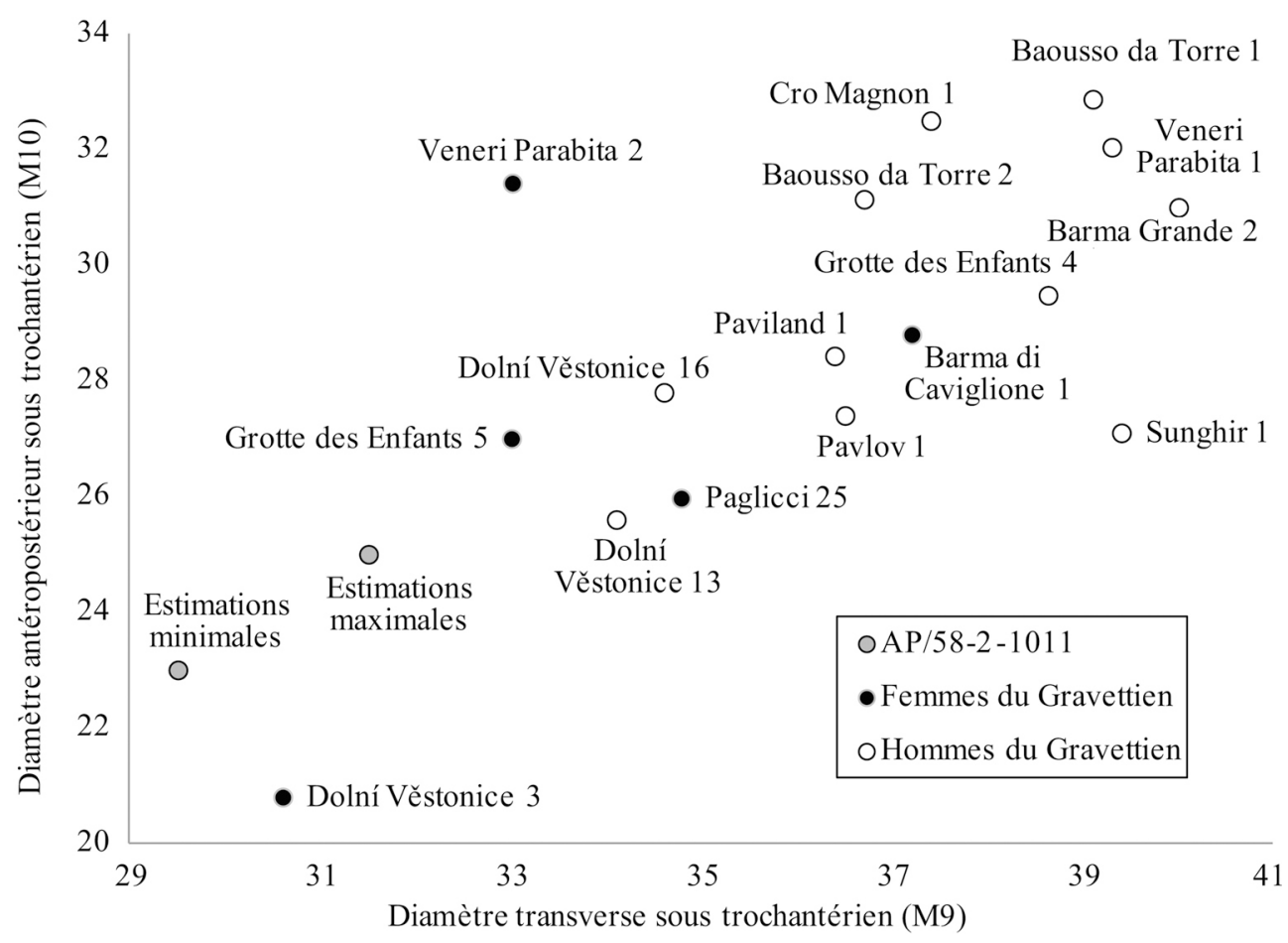

Fig. 11 Fémur, niveau sous trochantérien. Diamètre antéro-postérieur par rapport au diamètre transverse pour l'Abri Pataud et les autres fossiles du Gravettien (mesures du côté droit pour tous les individus sauf Grotte des Enfants 5 et Paviland 1) / Femur, subtrochanteric level. Anteroposterior versus transverse diameters for abri Pataud human remains and other Gravettian fossils (measures for the right side for all individuals but Grotte des Enfants 5, and Paviland 1).

Tableau 11 Mesures du premier métatarsien AP/--37801 et comparaison avec celles des os de P1 conservés au Musée de l'Homme / Measurements for the first metatarsal AP/-37801, and comparison with those of P1 curated in the Musée de l'Homme.

\begin{tabular}{|llll|}
\hline Individu & PX21 & \multicolumn{2}{c|}{ P1 } \\
\hline N $^{\circ}$ vestige & AP/--37801 & & \\
Côté & Gauche & Droit & Gauche \\
\hline Hauteur du corps (M4) & 12,1 & 13,5 & 13,2 \\
Largeur du corps (M3) & 13,6 & 11,8 & 11,3 \\
Hauteur proximale (M7) & $(26,7)$ & 26,6 & 26,7 \\
Largeur proximale (M6) & $(20,5)$ & & \\
\hline
\end{tabular}

métaphysaire) d'un pubis immature (dimension maximale : $15,1 \mathrm{~mm}$; Fig. 14d). L'identification de ce fragment n'est pas certaine, pas plus que son attribution à $\mathrm{P} 2$. En effet, ses dimensions sont relativement importantes par apport à l'ischium de P2, mais semblent un peu trop petites pour l'attribuer à $\mathrm{P} 6$.
Fémur droit (AP/89-2-37969)

Moitié proximale de la diaphyse, de la moitié de la zone d'insertion du muscle grand fessier à la mi-diaphyse environ (dimension maximale : 43,7 mm ; Fig. 14e).

La section de la diaphyse est ovoïde avec un grand axe antéro-postérieur (Tableau 13). La zone d'insertion du muscle grand fessier est irrégulière, avec l'aspect caractéristique des enthèses immatures. Le pilastre est très peu développé, à la surface légèrement irrégulière du côté médial. Deux foramens nourriciers sont présents postérieurement, l'un au niveau de la partie distale de l'insertion du muscle grand fessier, l'autre $11 \mathrm{~mm}$ plus bas. Cet os est le symétrique de fémur gauche du P2 (AP/63-1/2-1374) découvert en 1963.

\section{Tibia droit (AP/63-2-37953)}

Fragment de diaphyse immature, correspondant au tiers moyen environ (dimension maximale : 43,7 mm ; Fig. 14f).

Les mesures sont présentées dans le tableau 14. La section de la diaphyse est triangulaire. Un foramen est visible dans la partie proximale de la face postérieure. La morphologie générale de l'os est proche de celle du tibia gauche (AP/63-2-1409) attribué à P2. 


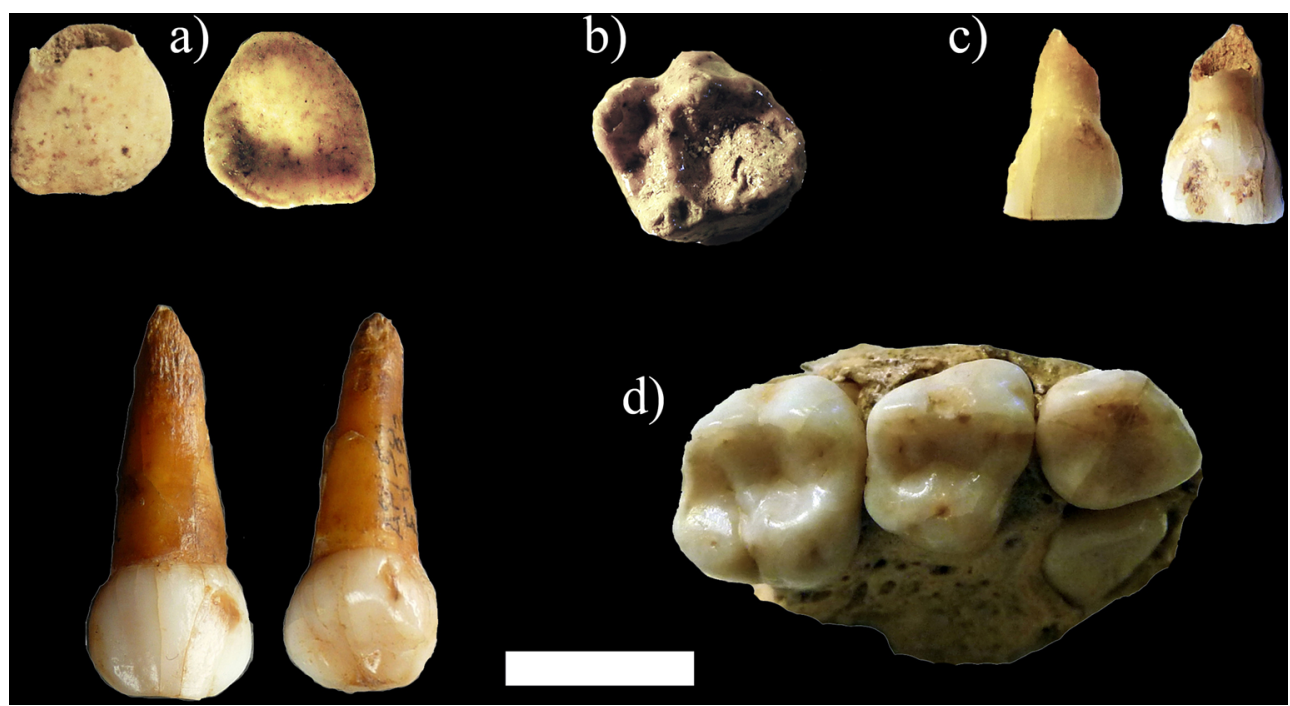

Fig. 12 Dents déciduales (échelle : $1 \mathrm{~cm}$ ). a : incisive latérale supérieure gauche (AP/10-2-W74A-1), faces vestibulaire et linguale, b : seconde molaire supérieure gauche (AP/89-2-72), c : incisive latérale supérieure gauche (AP/07-W76A-164-T550), d : canine supérieure droite (AP/58-2-F2283), faces vestibulaire et linguale, et remise en place dans le maxillaire attribué à P6 (clichés S. Villotte) / Deciduous teeth (scale: $1 \mathrm{~cm}$ ). a: upper left lateral incisor (AP/10-2-W74A-1), lingual and labial views, b: upper left second molar (AP/89-2-72), c: upper left lateral incisor (AP/07-W76A-164-T550), d: upper right canine (AP/58-2-F2283), lingual and labial views, and in the socket of the right maxilla of $P 6$ (Images: $S$. Villotte).

\section{Vestiges immatures attribués à P6}

\section{Dents}

Les mesures sont présentées dans le tableau 4.

\section{Canine déciduale supérieure droite (AP/58-2-F2283)}

Cette dent est complète (dimension maximale : $18,4 \mathrm{~mm}$ ), à l'apex fermé (Fig. 12d) (stade Ac dans [35]). L'extrémité de la racine est légèrement endommagée et a été recollée.

L'usure est faible, avec une exposition de la dentine sur une épaisseur mesurable (stade 3). Sur la face linguale, les crêtes mésiale et distale ainsi que le tubercule basal sont bien individualisés. Deux petites tâches marrons sont visibles sur la couronne, une sur la face vestibulaire du côté mésial, l'autre sur la face linguale du côté distal. Cette dent remonte parfaitement avec le maxillaire attribué à P6 (fig. 12d).

\section{Seconde incisive déciduale supérieure gauche (AP/07- 2-W76A-164-T550)}

Cette dent comprend la couronne et une partie de la racine (dimension maximale : 8,8 mm ; Fig. 12c). La couronne est en partie recouverte par du sédiment et par du tartre. Le bord incisif, rectiligne, est modérément usé (stade 3). La partie distale de la surface linguale présente une dépression assez marqué. La racine est en partie cassée et en partie résorbée. Le stade de résorption (R 1/2) suggère un âge entre 5 et 7 ans [35].

\section{Vertèbres}

Atlas (AP/58-2-37956)

Cet os, en deux fragments, est représenté par la masse latérale gauche, l'arc antérieur presque complet et une petite partie de l'arc postérieur (dimension maximale : 35,5 mm ; Fig. 13a). La cassure entre les deux fragments se situe postérieurement à la ligne de fusion entre l'arc antérieur et la masse latérale. La marge latérale des surfaces articulaires supérieure et inférieure est érodée. Le processus transverse gauche est manquant.

Les mesures sont présentées dans le tableau 5. La facette pour la dent est concave et ovoïde. L'arc antérieur et la masse latérale ne sont qu'en partie soudés, un espace est toujours présent antéro-supérieurement (la fusion s'opère généralement entre 5 et 6 ans [36]). La surface articulaire supérieure est large et concave, avec deux facettes mais sans séparation nette entre elles. La surface articulaire inférieure est plane. 


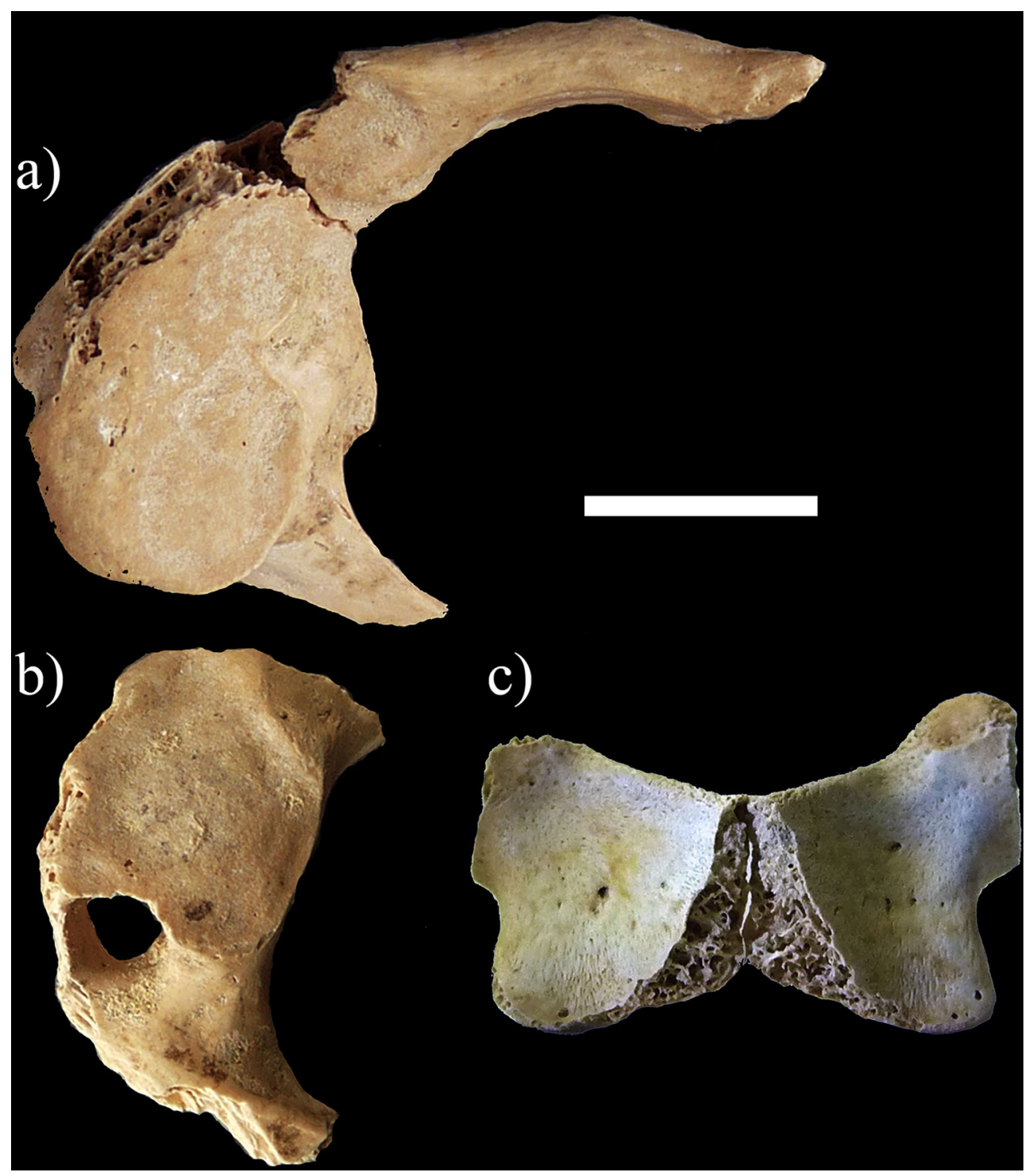

Fig. 13 Vertèbres immatures (échelle : $1 \mathrm{~cm})$. a : atlas (AP/58-2-37956). b : axis (AP/58-2-37957). c : hémi-arcs neuraux (AP/082-W76C-140). (clichés S. Villotte) / Immature vertebrae (scale : $1 \mathrm{~cm})$. a: atlas (AP/58-2-37956). b: axis (AP/58-2-37957). c: neural hemi-arches (AP/08-2-W76C-140). (Images: S. Villotte).

\section{Axis (AP/58-2-37957)}

Le fragment comprend l'arc neural gauche, la surface articulaire supérieure gauche (sa partie antérieure est manquante et sa marge latérale est érodée), le foramen transverse gauche et le tiers antérieur de la surface articulaire inférieure gauche (dimension maximale : 22,6 mm ; Fig. 13b).

La dent (manquante) semblait être fusionnée avec le corps alors que la synchondrose neurocentrale semblait toujours présente. Celle-ci se synostose entre 4 et 6 ans [36]. Cet os s'articule avec l'atlas AP/58-2-37956.

\section{Os des membres supérieurs}

\section{Scapula droite (AP/58-2-37952)}

Ce fragment correspond à la base de l'épine et à une portion triangulaire du corps d'une scapula immature (dimension maximale : 34,8 mm ; Fig. 14a).

Cet os présente peu de caractéristiques notables. A la jonction supérieure de l'épine et du corps, deux foramens sont présents latéralement. Un troisième, plus gros, est visible plus médialement. 


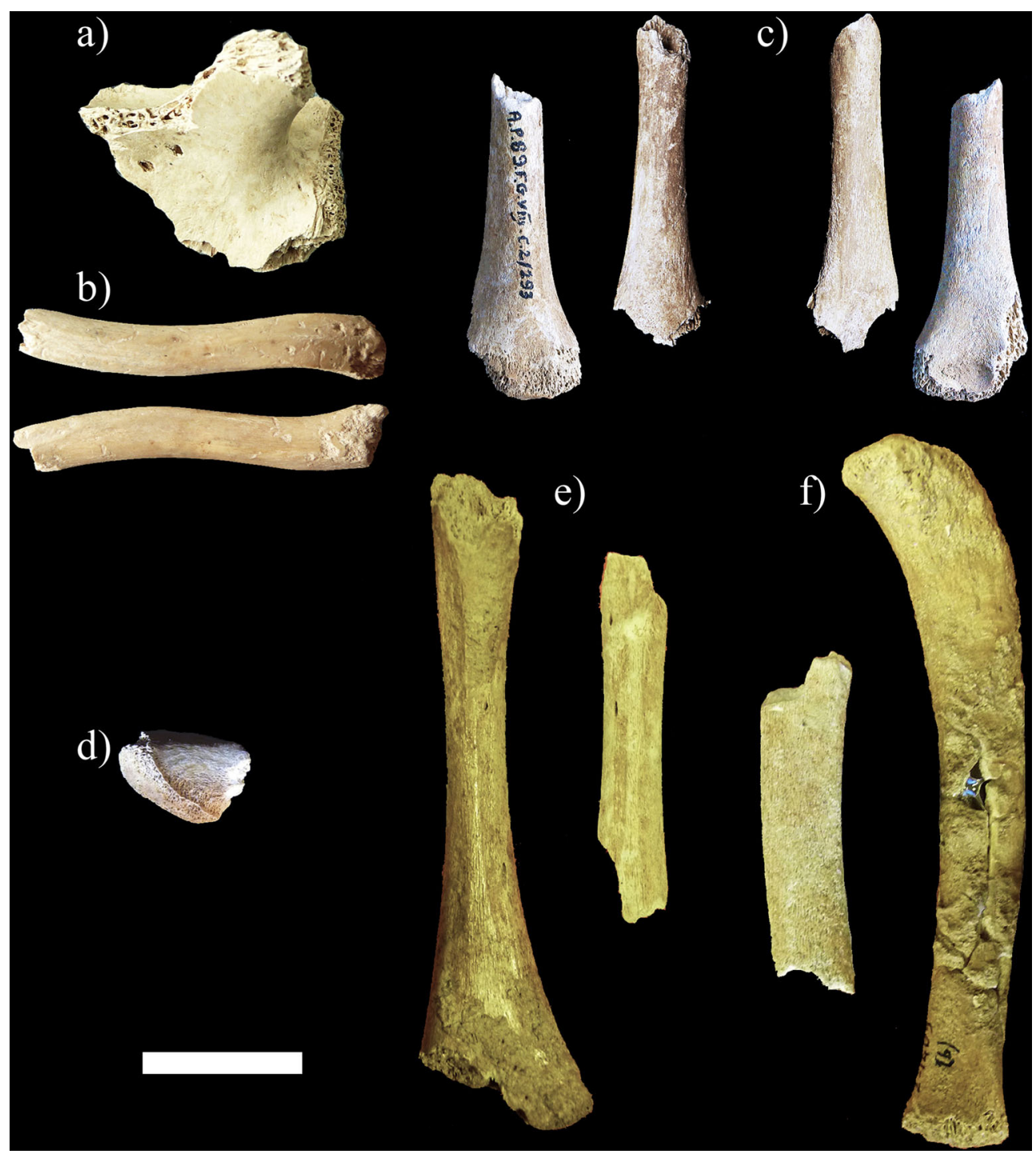

Fig. 14 Squelette appendiculaire immature (échelle : $2 \mathrm{~cm})$. a : scapula droite (AP/58-2-37952), b : clavicule gauche (AP/58-2-37959), vues supérieure et inférieure, $\mathrm{c}$ : humérus droits et gauche (AP/89-2-293 et AP/58-2-37955), vues antérieure et postérieure, $d$ : pubis gauche (?) (AP/63-2-37961), e : fémur droit (AP/89-2-37969) et son symétrique découvert en 1963, en vue postérieure, f : tibia droit (AP/632-37953) et son symétrique découvert en 1963, en vue médiale (clichés S. Villotte) / Immature appendicular skeleton (Scale: $2 \mathrm{~cm}$ ). a: right scapula (AP/58-2-37952), b: left clavicle (AP/58-2-37959), superior and inferior views, c: right and left humeri (AP/89-2-293 et AP/58-2-37955), anterior and posterior views, d: left pubis (?) (AP/63-2-37961), e: right femur (AP/89-2-37969), and the antimere discovered in 1963, posterior view, f: Right tibia (AP/63-2-37953), and the antimere discovered in 1963, medial view (Images: S. Villotte).

\section{Clavicule gauche (AP/58-2-37959)}

Fragment de diaphyse de clavicule immature, de la marge latérale de la zone d'insertion du muscle deltoïde au deux tiers de la diaphyse (dimension maximale : 46,4 $\mathrm{mm}$; Fig. 14b). Les dimensions sont présentées dans le tableau 10.

La clavicule est relativement droite. Sa face inférieure est plate à concave. La zone d'insertion pour le muscle deltoïde est bien distinguée, concave, avec une importante porosité.
L'estimation de la longueur totale (au moins $70 \mathrm{~mm}$ ) la place parmi les enfants de 3 à 5 ans.

\section{Discussion et conclusion}

Les vestiges humains présentés ici correspondent pour la plupart à des fragments de petites dimensions, ce qui limite leur étude. Par ailleurs, il s'agit essentiellement d'ossements 
Tableau 12 Mesures des humérus immatures AP/89-2-293 et $\mathrm{AP} / 58-2-37955$ / Measurements for the immature humeri AP/89-2-293 and AP/58-2-37955.

\begin{tabular}{|lll|}
\hline Individu & P2 \\
\hline $\begin{array}{l}\mathrm{N}^{\circ} \text { vestige } \\
\text { Côté }\end{array}$ & $\begin{array}{l}\text { AP/89-2-293 } \\
\text { Droit }\end{array}$ & $\begin{array}{l}\text { AP/58-2-37955 } \\
\text { Gauche }\end{array}$ \\
\hline $\begin{array}{l}\text { Diamètre antéro-postérieur } \\
\text { au trou nourricier }\end{array}$ & 6,5 & 7,1 \\
$\begin{array}{l}\text { Diamètre transverse } \\
\text { au trou nourricier } \\
\text { Périmètre au trou } \\
\text { nourricier }\end{array}$ & 6,5 & 6,1 \\
\hline
\end{tabular}

Tableau 13 Mesures du fémur immature AP/89-2-37969. La localisation de la mi diaphyse est estimée / Measurements for the immature femur AP/89-2-37969. Midshaft location is estimated.

\begin{tabular}{|ll|}
\hline Individu & P2 \\
\hline $\mathrm{N}^{\circ}$ vestige & $\mathrm{AP} / 89-$ \\
& $2-37969$ \\
Côté & Droit \\
\hline Diamètre transverse à mi diaphyse (M7) & 7,6 \\
Diamètre antéropostérieur à mi diaphyse (M6) & 8,9 \\
Circonférence à mi diaphyse (M8) & 26,5 \\
\hline
\end{tabular}

Tableau 14 Mesures du tibia immature AP/63-2-37953. La localisation de la mi diaphyse est estimée / Measurements for the immature tibia femur AP/63-2-37953. Midshaft location is estimated.

\begin{tabular}{|ll|}
\hline Individu & P2 \\
\hline $\mathrm{N}^{\circ}$ vestige & $\mathrm{AP} / 63-$ \\
& $2-37953$ \\
Côté & Droit \\
\hline Diamètre transverse à mi diaphyse (M9) & 7,2 \\
Diamètre antéro-postérieur à mi diaphyse & 8,8 \\
$(\mathrm{M} 8)$ & \\
Périmètre à mi diaphyse (M10) & 24,5 \\
Diamètre transverse au trou nourricier (M9a) & 7,4 \\
Diamètre antéro-postérieur au trou nourricier & 9,4 \\
$(\mathrm{M} 8 \mathrm{a})$ & \\
\hline
\end{tabular}

rarement décrits en détails en Anthropologie biologique (vertèbres, phalanges...) et les mesures qu'il est possible de prendre sur ces fossiles sont rarement publiées pour les autres vestiges gravettiens. Ainsi, peu de comparaisons pertinentes sont possibles.
De manière générale ces vestiges ne présentent pas de caractéristiques morphologiques notables, mis à part, pour les vestiges crâniens, la présence de nombreux foramens surnuméraires et la présence de cuspides surnuméraires pour les secondes prémolaires inférieures de $\mathrm{P} 1$.

Les os attribués à $\mathrm{P} 1$ sont de petites dimensions, contrairement à ses dents qui sont de dimensions moyennes à grandes (surtout pour les diamètres vestibulo-linguaux) pour la période. Les grandes dimensions dentaires et le nombre important de cuspides avaient déjà été remarqués pour les autres dents de P1 par Legoux [11]. AP/58-2-1011 est le seul fragment de fémur adulte retrouvé pour la couche 2 . Il s'agit d'un fragment ocré [6]. Or, tous les autres vestiges humains ocrés de la couche 2 sont très certainement attribuables à $\mathrm{P} 1$ [6]. Ce point et le fait que ce fémur est de petites dimensions plaident également en faveur d'une attribution à P1. Parmi les autres vestiges adultes, certains présentent des caractéristiques (taille et forme) qui permettent de les associer avec une certaine assurance à P5. Étonnamment, il s'agit uniquement d'os des membres supérieurs : la scapula gauche (AP/58-2-592), les clavicules gauche (AP/58-2-F1026A) et droite (AP/58-2-F1026B), et le métacarpien III droit (AP/582-37964). P5 serait donc, tout comme P3, uniquement représenté par des os des deux membres supérieurs (notons cependant qu'un fragment de fibula découvert en 1958, assez robuste, pourrait lui appartenir [6]). Ces résultats n'invalident pas ceux avancés précédemment [6], mais permettent ainsi de préciser certaines attributions. Les ossements maintenant attribués à chacun des six individus sont listés dans les tableaux 15 à 20.

Les travaux récents sur les fossiles de la couche 2 [ 6 et cet article], et sur leur contexte culturel [2-5] témoignent de leur importance, que l'on s'intéresse à la biologie ou aux comportements des groupes humains du Gravettien. Ces travaux constituent une première étape de révision de tous les vestiges issus de la couche 2 et appellent une ré-analyse plus détaillée des caractéristiques biologiques. En effet, depuis leur première description dans les années soixante dix [811], de nouvelles découvertes et de nouveaux paradigmes ont permis de reformuler les hypothèses sur le peuplement au Paléolithique supérieur en Europe. En raison de leur position chronologique (28000-26000 cal. BP), les squelettes de l'abri Pataud constituent notamment un élément clef pour la discussion des modèles de microévolutions qui définissent au moins deux phases distinctes durant le Paléolithique supérieur, avant et après le dernier maximum glaciaire [38].

Remerciements Nous remercions Aurélie Fort, Véronique Laborde et Liliana Huet (MNHN, Musée de l'Homme) pour leur accueil et leur disponibilité lors des études des vestiges issus des fouilles Movius, Laurent Crépin (MNHN) pour les nombreuses discussions sur la taphonomie et l'identification des vestiges humains, et Asier Gó 
Tableau 15 Vestiges humains attribués à P1 d'après [6] et cette étude. Le numéro unique (première colonne) n'étant pas marqué sur les pièces conservées au Musée de l'Homme ( $\mathrm{MH}$ ), d'autres indications (numéros de boite, inscriptions, numéros utilisés dans [8] et [11]) sont également fournies pour faciliter leur identification / Human remains attributed to P1 after [6] and the present study. The unique number (in the first column) has never been marked on the bones curated at the Musée de l'Homme (MH), and other identifiers (box number, labels, numbers used in [8] and [11]) are also supplied to facilitate their identification.

\begin{tabular}{|c|c|c|c|c|}
\hline $\mathbf{N}^{\circ}$ & Vestiges & $\mathrm{N}^{\circ}$ Boite MH & $\mathbf{N}^{\circ} \mathbf{M H}$ & Description bio. \\
\hline $\mathrm{AP} / 58-2-37806$ & Bloc craniofacial et dents & 9 & 26224 & {$[8]$ et $[11]\left(n^{\circ} 2\right)$} \\
\hline $\mathrm{AP} / 58-2-37807$ & Mandibule et dents & 10 & 26224 & {$[8]$ et $[11]\left(n^{\circ} 2\right)$} \\
\hline $\mathrm{AP} / 89-2-37968$ & Hyoïde & & & Cette étude \\
\hline $\mathrm{AP} / 89-2-67$ & Incisive 1 ou $2 \operatorname{Inf}$ & & & Cette étude \\
\hline $\mathrm{AP} / 89-2-63$ & Incisive 1 ou $2 \operatorname{Inf}$ & & & Cette étude \\
\hline AP/89-2-62 & Incisive 1 Sup droite & & & Cette étude \\
\hline $\mathrm{AP} / 89-2-71$ & Incisive 1 Sup gauche & & & Cette étude \\
\hline AP/89-2-66 & Incisive 2 Sup droite & & & Cette étude \\
\hline $\mathrm{AP} / 63-2-2088$ & Incisive 2 Sup gauche & 64 & 26224 bis & {$[11]\left(n^{\circ} 2088\right)$} \\
\hline AP/89-2-64 & Canine Inf droite & & & Cette étude \\
\hline $\mathrm{AP} / 89-2-70$ & Canine Inf gauche & & & Cette étude \\
\hline AP/89-2-61 & Canine Sup droite & & & Cette étude \\
\hline $\mathrm{AP} / 89-2-68$ & Canine Sup gauche & & & Cette étude \\
\hline $\mathrm{AP} / 63-2-1715$ & Prémolaire 1 Sup droite & 64 & 26224 bis & {$[11]\left(\mathrm{n}^{\circ} 1715\right)$} \\
\hline AP/89-2-69 & Prémolaire 2 Inf droite & & & Cette étude \\
\hline $\mathrm{AP} / 89-2-65$ & Prémolaire 2 Inf gauche & & & Cette étude \\
\hline $\mathrm{AP} / 63-2-2087$ & Prémolaire 2 Sup droite & 64 & 26224 bis & {$[11]\left(\mathrm{n}^{\circ} 2087\right)$} \\
\hline $\mathrm{AP} / 63-2-2089$ & Dent surn. droite & 64 & 26224 bis & {$[11]\left(n^{\circ} 2089\right)$} \\
\hline AP/89-2-309 & Vertèbre Cervicale 1 (4 fragments) & & & Cette étude \\
\hline $\mathrm{AP} / 63-1 / 2-1397 \mathrm{~B}$ & Vertèbre Cervicale 2 & 64 & 26225 & {$[8]\left(n^{\circ} 1397 B\right)$} \\
\hline $\mathrm{AP} / 63-2-1460$ & Vertèbre Cervicale 3 & 64 & 26225 & {$[8]\left(n^{\circ} 1460\right)$} \\
\hline $\mathrm{AP} / 63-1 / 2-1366$ & Vertèbre Cervicale 4 & 64 & 26225 & {$[8]\left(\mathrm{n}^{\circ} 1366\right)$} \\
\hline AP/89-2-37979 & Vertèbre Cervicale 5 ou 6 & & & Cette étude \\
\hline $\mathrm{AP} / 63-1 / 2-1380$ & Vertèbre Cervicale 7 & 64 & 26225 & {$[8]\left(n^{\circ} 1380\right)$} \\
\hline AP/89-2-37975 & Vertèbre Cervicale ou Thoracique & & & Cette étude \\
\hline $\mathrm{AP} / 89-2-37976$ & Vertèbre Thoracique & & & Cette étude \\
\hline $\mathrm{AP} / 89-2-37967$ & Vertèbre Thoracique & & & Cette étude \\
\hline $\mathrm{AP} / 89-2-37978$ & Vertèbre Thoracique & & & Cette étude \\
\hline $\mathrm{AP} / 89-2-37980$ & Vertèbre Thoracique & & & Cette étude \\
\hline $\mathrm{AP} / 89-2-313$ & Vertèbre Thoracique & & & Cette étude \\
\hline AP/89-2-288 & Vertèbre Thoracique & & & Cette étude \\
\hline $\mathrm{AP} / 89-2-37974$ & Vertèbre Thoracique Haute & & & Cette étude \\
\hline AP/63-1/2-1396 & Vertèbre Thoracique 3 ou 4 & 64 & 26225 & {$[8]\left(n^{\circ} 1396\right)$} \\
\hline $\mathrm{AP} / 63-1 / 2-1400$ & Vertèbre Thoracique 4 à 6 & 64 & 26225 & {$[8]\left(\mathrm{n}^{\circ} 1400\right)$} \\
\hline $\mathrm{AP} / 63-1 / 2-1397 \mathrm{~A}$ & Vertèbre Thoracique 5 à 7 & 64 & 26225 & [8] $\left(\mathrm{n}^{\circ} 1397 \mathrm{~A}\right)$ \\
\hline $\mathrm{AP} / 63-1 / 2-1406$ & Vertèbre Thoracique 6 à 8 & 64 & 26225 & {$[8]\left(n^{\circ} 1406\right)$} \\
\hline $\mathrm{AP} / 63-1 / 2-1375$ & Vertèbre Thoracique 9 ou 10 & 64 & 26225 & {$[8]\left(\mathrm{n}^{\circ} 1375\right)$} \\
\hline $\mathrm{AP} / 89-2-37977$ & Vertèbre Thoracique $11 ?$ & & & Cette étude \\
\hline $\mathrm{AP} / 89-2-310$ & Vertèbre Thoracique $12 ?$ & & & Cette étude \\
\hline $\mathrm{AP} / 63-1 / 2-1382$ & Vertèbre Lombaire 2 ou 3 & 64 & 26225 & {$[8]\left(\mathrm{n}^{\circ} 1382\right)$} \\
\hline $\mathrm{AP} / 63-2-1304$ & Vertèbre Lombaire 3 ou 4 & 64 & 26225 & {$[8]\left(n^{\circ} 1304\right)$} \\
\hline AP/63-1/2-1405 & Côte & 64 & 26226 & {$[8]\left(\mathrm{n}^{\circ} 1405\right)$} \\
\hline $\mathrm{AP} / 63-1 / 2-1367$ & Côte & 64 & 26226 & $\begin{array}{l}{[8]\left(\mathrm{n}^{\circ} 1367\right)} \\
\quad \text { (Suite page suivante) }\end{array}$ \\
\hline
\end{tabular}




\begin{tabular}{|c|c|c|c|c|}
\hline $\mathbf{N}^{\circ}$ & Vestiges & $\mathbf{N}^{\circ}$ Boite MH & $\mathbf{N}^{\circ} \mathbf{M H}$ & Description bio. \\
\hline $\mathrm{AP} / 63-2-1459$ & Côte & 64 & 26226 & {$[8]\left(n^{\circ} 1459\right)$} \\
\hline $\mathrm{AP} / 63-1 / 2-1312$ & Côte (6 fragments) & 64 & 26226 & {$[8]\left(\mathrm{n}^{\circ} 1312\right)$} \\
\hline AP/89-2-37973 & Côte droite 1 & & & Cette étude \\
\hline $\mathrm{AP} / 63-1 / 2-1365$ & Côte droite 3 ou 4 & 64 & 26226 & {$[8]\left(\mathrm{n}^{\circ} 1365\right)$} \\
\hline $\mathrm{AP} / 63-1 / 2-1384$ & Côte droite 4 à 6 & 64 & 26226 & {$[8]\left(n^{\circ} 1384\right)$} \\
\hline $\mathrm{AP} / 63-1 / 2-1373$ & Côte droite 7 ou 8 & 64 & 26226 & {$[8]\left(\mathrm{n}^{\circ} 1373\right)$} \\
\hline $\mathrm{AP} / 63-2-1311$ & Côte droite 11 & 64 & 26226 & {$[8]\left(\mathrm{n}^{\circ} 1311\right)$} \\
\hline $\mathrm{AP} / 63-1 / 2-1377$ & Côte gauche 2 (7 fragments) & 64 & 26226 & {$[8]\left(\mathrm{n}^{\circ} 1377\right)$} \\
\hline $\mathrm{AP} / 63-2-1514$ & Côte gauche 8 ou 9 & 64 & 26226 & {$[8]\left(n^{\circ} 1514\right)$} \\
\hline $\mathrm{AP} / 63-2-1511$ & Côte gauche 10 & 64 & 26226 & {$[8]\left(\mathrm{n}^{\circ} 1511\right)$} \\
\hline $\mathrm{AP} / 63-2-1535$ & Côte gauche 11 & 62 & 1535 & $?$ \\
\hline AP/89-2-299 & Côte gauche 12 & & & Cette étude \\
\hline $\mathrm{AP} / 63-1 / 2-1379$ & Côte gauche ? 10 ou 11 & 64 & 26226 & {$[8]\left(n^{\circ} 1379\right)$} \\
\hline $\mathrm{AP} / 63-2-1513$ & Clavicule droite & 64 & 26229 & {$[8]\left(\mathrm{n}^{\circ} 1513\right)$} \\
\hline $\mathrm{AP} / 63-2-1519$ & Humérus gauche & 64 & 26229 & {$[8]\left(\mathrm{n}^{\circ} 1519\right)$} \\
\hline $\mathrm{AP} / 63-2-37960$ & Scaphoïde droit & & & Cette étude \\
\hline $\mathrm{AP} / 89-2-37970$ & Trapézoïde droit & & & Cette étude \\
\hline $\mathrm{AP} / 63-2-1541$ & Lunatum droit & 64 & 26227 & {$[8]\left(\mathrm{n}^{\circ} 1541\right)$} \\
\hline $\mathrm{AP} / 63-2-2086$ & Capitatum droit & 64 & 26227 & {$[8]\left(n^{\circ} 2086\right)$} \\
\hline $\mathrm{AP} / 63-2-1832$ & Hamatum droit & 64 & 26227 & {$[8]\left(\mathrm{n}^{\circ} 1832\right)$} \\
\hline $\mathrm{AP} / 63-2-1714$ & Triquetrum droit & 64 & 26227 & {$[8]\left(n^{\circ} 1714\right)$} \\
\hline $\mathrm{AP} / 63-2-1540$ & Pisiforme droit & 64 & 26227 & {$[8]\left(\mathrm{n}^{\circ} 1540\right)$} \\
\hline AP/63-2-2091 & Scaphoïde gauche & 64 & 26227 & {$[8]\left(n^{\circ} 2091\right)$} \\
\hline $\mathrm{AP} / 63-1 / 2-1385$ & Trapèze gauche & 64 & 26227 & {$[8]\left(\mathrm{n}^{\circ} 1385\right)$} \\
\hline $\mathrm{AP} / 63-2-1510$ & Lunatum gauche & 64 & 26227 & {$[8]\left(n^{\circ} 1510\right)$} \\
\hline $\mathrm{AP} / 63-2-1716$ & Triquetrum gauche & 64 & 26227 & {$[8]\left(\mathrm{n}^{\circ} 1716\right)$} \\
\hline $\mathrm{AP} / 63-2-2085$ & Capitatum gauche & 64 & 26227 & {$[8]\left(n^{\circ} 2085\right)$} \\
\hline AP/63-2-1539 & Hamatum gauche & 64 & 26227 & [8] $\left(\mathrm{n}^{\circ} 1539\right)$ \\
\hline $\mathrm{AP} / 63-2-1516$ & MTC droit 1 & 64 & 26227 & {$[8]\left(n^{\circ} 1516\right)$} \\
\hline $\mathrm{AP} / 63-1 / 2-1404$ & MTC droit 4 & 64 & 26227 & {$[8]\left(n^{\circ} 1404\right)$} \\
\hline $\mathrm{AP} / 89-2-312$ & MTC droit 5 ? & & & Cette étude \\
\hline $\mathrm{AP} / 63-2-1538$ & MTC gauche 1 & 64 & 26227 & {$[8]\left(\mathrm{n}^{\circ} 1538\right)$} \\
\hline $\mathrm{AP} / 63-2-2081$ & MTC gauche 2 & 64 & 26227 & {$[8]\left(\mathrm{n}^{\circ} 2081\right)$} \\
\hline $\mathrm{AP} / 63-2-1521$ & MTC gauche 3 & 64 & 26227 & {$[8]\left(n^{\circ} 1521\right)$} \\
\hline AP/89-2-306 & MTC gauche 4 & & & Cette étude \\
\hline $\mathrm{AP} / 89-2-37971$ & MTC gauche ? 4? & & & Cette étude \\
\hline $\mathrm{AP} / 63-2-1520$ & Phalange prox. droite 1 Main & 64 & 26227 & {$[8]\left(n^{\circ} 1520\right)$} \\
\hline $\mathrm{AP} / 63-2-1536$ & Phalange prox. droite 2 Main & 64 & 26227 & {$[8]\left(\mathrm{n}^{\circ} 1536\right)$} \\
\hline $\mathrm{AP} / 63-2-1518$ & Phalange prox. droite 3 Main & 64 & 26227 & {$[8]\left(n^{\circ} 1518\right)$} \\
\hline AP/63-2-1532 & Phalange prox. droite 4 Main & 64 & 26227 & {$[8]\left(\mathrm{n}^{\circ} 1532\right)$} \\
\hline $\mathrm{AP} / 63-1 / 2-1313$ & Phalange prox. droite 5 Main & 64 & 26227 & {$[8]\left(n^{\circ} 1313\right)$} \\
\hline AP/63-2-2077 & Phalange prox. gauche 2 Main & 64 & 26227 & {$[8]\left(n^{\circ} 2077\right)$} \\
\hline $\mathrm{AP} / 63-2-1530$ & Phalange prox. gauche 3 Main & 64 & 26227 & {$[8]\left(\mathrm{n}^{\circ} 1530\right)$} \\
\hline AP/89-2-302 & Phalange prox. gauche 4 Main & & & Cette étude \\
\hline $\mathrm{AP} / 63-2-1711$ & Phalange prox. gauche 5 Main & 64 & 26227 & {$[8]\left(\mathrm{n}^{\circ} 1711\right)$} \\
\hline $\mathrm{AP} / 63-1 / 2-1370$ & Phalange méd. droite 2 Main & 64 & 26227 & {$[8]\left(n^{\circ} 1370\right)$} \\
\hline $\mathrm{AP} / 63-1 / 2-1368$ & Phalange méd. droite 3 Main & 64 & 26227 & {$[8]\left(n^{\circ} 1368\right)$} \\
\hline
\end{tabular}




\begin{tabular}{|c|c|c|c|c|}
\hline $\mathbf{N}^{\circ}$ & Vestiges & $\mathbf{N}^{\circ}$ Boite MH & $\mathbf{N}^{\circ} \mathbf{M H}$ & Description bio. \\
\hline $\mathrm{AP} / 63-2-1534$ & Phalange méd. droite 4 Main & 64 & 26227 & {$[8]\left(n^{\circ} 1534\right)$} \\
\hline $\mathrm{AP} / 63-2-1542$ & Phalange méd. droite 5 Main & 64 & 26227 & {$[8]\left(\mathrm{n}^{\circ} 1542\right)$} \\
\hline $\mathrm{AP} / 63-2-1712$ & Phalange méd. gauche 2 Main & 64 & 26227 & {$[8]\left(n^{\circ} 1712\right)$} \\
\hline $\mathrm{AP} / 63-1 / 2-1402$ & Phalange méd. gauche 4 Main & 64 & 26227 & {$[8]\left(\mathrm{n}^{\circ} 1402\right)$} \\
\hline $\mathrm{AP} / 63-1 / 2-37962$ & Phalange méd. gauche 5 ? Main & & & Cette étude \\
\hline $\mathrm{AP} / 63-2-1829$ & Phalange dist. droite 1 Main & 64 & 26227 & {$[8]\left(n^{\circ} 1829\right)$} \\
\hline $\mathrm{AP} / 63-2-2069$ & Phalange dist. droite 2 Main & 64 & 26227 & {$[8]\left(n^{\circ} 2069\right)$} \\
\hline $\mathrm{AP} / 63-2-2095$ & Phalange dist. droite 3 Main & 64 & 26227 & {$[8]\left(n^{\circ} 2095\right)$} \\
\hline $\mathrm{AP} / 63-2-1537$ & Phalange dist. droite 4 Main & 64 & 26227 & {$[8]\left(\mathrm{n}^{\circ} 1537\right)$} \\
\hline $\mathrm{AP} / 63-2-1833$ & Phalange dist. droite 5 Main & 64 & 26227 & {$[8]\left(\mathrm{n}^{\circ} 1833\right)$} \\
\hline AP/89-2-465 & Phalange dist. gauche 5 Main & & & Cette étude \\
\hline $\mathrm{AP} / 63-2-1464$ & Coxal droit & 63 & 26235 & [8] $\left(\right.$ Pas de $\left.n^{\circ}\right)$ \\
\hline $\mathrm{AP} / 58-2-37808$ & Coxal gauche & 63 & 26235 & [8] $\left(\right.$ Pas de $\left.n^{\circ}\right)$ \\
\hline $\mathrm{AP} / 58-2-2 \mathrm{~F} 1011$ & Fémur droit & & & Cette étude \\
\hline $\mathrm{AP} / 63-1 / 2-1376$ & Patella droite & 64 & 26229 & {$[8]\left(n^{\circ} 1376\right)$} \\
\hline AP/63-2-1543 & Patella gauche & 64 & 26229 & {$[8]\left(n^{\circ} 1543\right)$} \\
\hline AP/63-1/2-1381 & Talus droit & 64 & 26228 & {$[8]\left(n^{\circ} 1381\right)$} \\
\hline AP/63-1/2-1408 & Naviculaire droit & 64 & 26228 & {$[8]\left(n^{\circ} 1408\right)$} \\
\hline $\mathrm{AP} / 63-1 / 2-1331$ & Cuboïde droit & 64 & 26228 & {$[8]\left(\mathrm{n}^{\circ} 1331\right)$} \\
\hline AP/63-2-1463 & Cunéiforme médial droit & 64 & 26228 & {$[8]\left(n^{\circ} 1463\right)$} \\
\hline $\mathrm{AP} / 63-2-1307$ & Cunéiforme latéral droit & 64 & 26228 & {$[8]\left(\mathrm{n}^{\circ} 1307\right)$} \\
\hline $\mathrm{AP} / 63-1 / 2-1407$ & Calcaneus gauche & 64 & 26228 & {$[8]\left(n^{\circ} 1407\right)$} \\
\hline $\mathrm{AP} / 63-1 / 2-1330$ & Talus gauche & 64 & 26228 & {$[8]\left(n^{\circ} 1330\right)$} \\
\hline $\mathrm{AP} / 63-1 / 2-1399$ & Naviculaire gauche & 64 & 26228 & {$[8]\left(\mathrm{n}^{\circ} 1399\right)$} \\
\hline AP/63-2-1512 & Cuboïde gauche & 64 & 26228 & {$[8]\left(n^{\circ} 1512\right)$} \\
\hline $\mathrm{AP} / 63-2-2090$ & Cunéiforme intér. gauche & 64 & 26228 & {$[8]\left(n^{\circ} 2090\right)$} \\
\hline $\mathrm{AP} / 63-1 / 2-1333$ & Cunéiforme latéral gauche & 64 & 26228 & {$[8]\left(n^{\circ} 1333\right)$} \\
\hline $\mathrm{AP} / 63-2-1309$ & MTT droit 1 & 64 & 26228 & {$[8]\left(\mathrm{n}^{\circ} 1309\right)$} \\
\hline $\mathrm{AP} / 63-1 / 2-1327$ & MTT droit 2 & 64 & 26228 & {$[8]\left(n^{\circ} 1327\right)$} \\
\hline $\mathrm{AP} / 63-2-1310$ & MTT droit 3 & 64 & 26228 & {$[8]\left(\mathrm{n}^{\circ} 1310\right)$} \\
\hline $\mathrm{AP} / 89-2-37972$ & MTT droit 4 ? & & & Cette étude \\
\hline AP/63-1/2-1398 & MTT droit 5 & 64 & 26228 & {$[8]\left(n^{\circ} 1398\right)$} \\
\hline $\mathrm{AP} / 63-1 / 2-1326$ & MTT gauche 1 & 64 & 26228 & {$[8]\left(\mathrm{n}^{\circ} 1326\right)$} \\
\hline AP/63-1/2-1395 & MTT gauche 2 & 64 & 26228 & {$[8]\left(n^{\circ} 1395\right)$} \\
\hline $\mathrm{AP} / 63-2-1305$ & MTT gauche 3 & 64 & 26228 & {$[8]\left(n^{\circ} 1305\right)$} \\
\hline AP/63-1/2-1332 & MTT gauche 4 & 64 & 26228 & {$[8]\left(n^{\circ} 1332\right)$} \\
\hline $\mathrm{AP} / 63-1 / 2-1329$ & MTT gauche 5 & 64 & 26228 & {$[8]\left(n^{\circ} 1329\right)$} \\
\hline AP/63-2-1465 & Phalange prox. droite 1 Pied & 64 & 26228 & {$[8]\left(n^{\circ} 1465\right)$} \\
\hline $\mathrm{AP} / 63-1 / 2-1386$ & Phalange prox. droite 2 Pied & 64 & 26228 & {$[8]\left(\mathrm{n}^{\circ} 1386\right)$} \\
\hline AP/63-2-1306 & Phalange prox. droite 3 Pied & 64 & 26228 & {$[8]\left(n^{\circ} 1306\right)$} \\
\hline AP/63-2-1462 & Phalange prox. droite 4 Pied & 64 & 26228 & {$[8]\left(n^{\circ} 1462\right)$} \\
\hline $\mathrm{AP} / 63-1 / 2-1389$ & Phalange prox. droite 5 Pied & 64 & 26228 & {$[8]\left(n^{\circ} 1389\right)$} \\
\hline AP/63-1/2-1403 & Phalange prox. gauche 1 Pied & 64 & 26228 & {$[8]\left(n^{\circ} 1403\right)$} \\
\hline AP/63-2-1461 & Phalange prox. gauche 2 Pied & 64 & 26228 & {$[8]\left(\mathrm{n}^{\circ} 1461\right)$} \\
\hline AP/63-1/2-1387 & Phalange prox. gauche 3 Pied & 64 & 26228 & {$[8]\left(n^{\circ} 1387\right)$} \\
\hline AP/63-1/2-1388 & Phalange prox. gauche 4 Pied & 64 & 26228 & {$[8]\left(n^{\circ} 1388\right)$} \\
\hline $\mathrm{AP} / 63-2-1467$ & Phalange prox. gauche 5 Pied & 64 & 26228 & {$[8]\left(n^{\circ} 1467\right)$} \\
\hline $\mathrm{AP} / 63-2-1466$ & Phalange dist. droite 1 Pied & 64 & 26228 & {$[8]\left(\mathrm{n}^{\circ} 1466\right)$} \\
\hline $\mathrm{AP} / 63-1 / 2-1334$ & Phalange dist. gauche 1 Pied & 64 & 26228 & {$[8]\left(\mathrm{n}^{\circ} 1334\right)$} \\
\hline
\end{tabular}




\begin{tabular}{|c|c|c|c|c|}
\hline $\mathbf{N}^{\circ}$ & Vestiges & $\mathbf{N}^{\circ}$ Boite MH & $\mathbf{N}^{\circ} \mathbf{M H}$ & Description bio. \\
\hline $\mathrm{AP} / 63-1 / 2-1401$ & Mandibule & 67 & 26234B & {$[8]\left(\mathrm{n}^{\circ} 1401\right)$} \\
\hline AP/10-2-W74A-1 & Incisive déciduale 2 Sup gauche & & & Cette étude \\
\hline $\mathrm{AP} / 89-2-72$ & Molaire déciduale 2 Sup gauche & & & Cette étude \\
\hline $\mathrm{AP} / 08-2-\mathrm{W} 76 \mathrm{C}-140$ & Vertèbre Lombaire & & & Cette étude \\
\hline AP/63-2-1531 & Côte & 67 & 26234B & {$[8]\left(n^{\circ} 1531\right)$} \\
\hline $\mathrm{AP} / 63-2-1517$ & Côte & 67 & 26234B & {$[8]\left(\mathrm{n}^{\circ} 1517\right)$} \\
\hline $\mathrm{AP} / 63-2-2092$ & Côte & 67 & 26234B & {$[8]\left(\mathrm{n}^{\circ} 2092\right)$} \\
\hline $\mathrm{AP} / 63-2-1713$ & Côte droite & 67 & 26234B & {$[8]\left(\mathrm{n}^{\circ} 1713\right)$} \\
\hline $\mathrm{AP} / 63-2-1830$ & Côte droite & 67 & 26234B & {$[8]\left(\mathrm{n}^{\circ} 1830\right)$} \\
\hline $\mathrm{AP} / 63-2-1515$ & Côte droite & 67 & 26234B & {$[8]\left(\mathrm{n}^{\circ} 1515\right)$} \\
\hline $\mathrm{AP} / 63-2-1828$ & Côte gauche & 67 & 26234B & {$[8]\left(n^{\circ} 1828\right)$} \\
\hline $\mathrm{AP} / 63-1 / 2-1371$ & Côte gauche & 67 & 26234B & {$[8]\left(\mathrm{n}^{\circ} 1371\right)$} \\
\hline $\mathrm{AP} / 63-1 / 2-1383$ & Côte gauche & 67 & 26234B & {$[8]\left(n^{\circ} 1383\right)$} \\
\hline AP/63-2-1533 & Côte gauche & 67 & 26234B & {$[8]\left(n^{\circ} 1533\right)$} \\
\hline AP/89-2-293 & Humérus droit & & & Cette étude \\
\hline $\mathrm{AP} / 58-2-37955$ & Humérus gauche & & & Cette étude \\
\hline $\mathrm{AP} / 63-1 / 2-1372$ & Coxal gauche (ischium) & 67 & 26234B & {$[8]\left(\mathrm{n}^{\circ} 1372\right)$} \\
\hline $\mathrm{AP} / 63-2-37961$ & Coxal gauche (pubis)? & & & Cette étude \\
\hline $\mathrm{AP} / 89-2-37969$ & Fémur droit & & & Cette étude \\
\hline $\mathrm{AP} / 63-1 / 2-1374$ & Fémur gauche & 67 & 26234B & {$[8]\left(n^{\circ} 1374\right)$} \\
\hline AP/63-2-37953 & Tibia droit & & & Cette étude \\
\hline AP/63-2-1409 & Tibia gauche & 67 & 26234B & {$[8]\left(n^{\circ} 1409\right)$} \\
\hline $\mathrm{AP} / 63-2-2093$ & MTT droit 1 & 67 & 26234B & {$[8]\left(n^{\circ} 2093\right)$} \\
\hline AP/63-2-1717 & MTT gauche 1 & 67 & $26234 \mathrm{~B}$ & {$[8]\left(n^{\circ} 1717\right)$} \\
\hline
\end{tabular}

mez-Olivencia (Université du Pays basque) pour avoir aidé à l'identification de certains vestiges rachidiens. Nous remercions également les conservateurs des différents musées ayant permis l'acquisition de données de comparaison ou l'accès aux archives (Musée d'Archéologie Nationale, Musée de Nancy, Musée National de Préhistoire, musée d'Anthropologie préhistorique de Monaco), ainsi que Jaroslav Brůžek (PACEA), Erik Trinkaus (Washington University in St. Louis), et Trenton Holliday (Tulane University) qui nous ont fourni des données non publiées pour certains fossiles. Enfin, nous remercions les deux relecteurs anonymes pour leurs remarques.
L'opération archéologique programmée « L'occupation humaine de l'abri Pataud il y a 22000 ans (Les Eyziesde-Tayac, Dordogne) » a bénéficié du soutien financier du Service régional d'archéologie d'Aquitaine, du Conseil général de la Dordogne, de l'UMR 7194 « Histoire naturelle de l'homme préhistorique », du département de Préhistoire du Muséum national d'histoire naturelle, ainsi que des programmes financés par l'ANR « MADAPCA » (2008-2011, ANR-07-BLAN-0011, coordination Patrick Paillet), « GUEROPE »(2007-2010, ANR-06-CONF-0008-01, coordination Luc Baray) et "ArBoCo » (2007-2010, ANR07-JCJCC-0149, coordination Ina Reiche). 
Tableau 17 Vestiges humains attribués à P3 d'après [6] et cette étude. Cf. Légende du tableau 15 / Human remains attributed to P3 after [6] and the present study. Cf. caption of table 15.

\begin{tabular}{|c|c|c|c|c|}
\hline $\mathbf{N}^{\circ}$ & Vestiges & $\mathbf{N}^{\circ}$ Boite MH & $\mathbf{N}^{\circ} \mathbf{M H}$ & Description bio. \\
\hline $\mathrm{AP} / 58-2-37832$ & Scapula droite & 68 & $26230 \mathrm{~A}$ & {$[8]\left(n^{\circ} 22\right)$} \\
\hline $\mathrm{AP} / 58-2-37830$ & Clavicule droite & 68 & $26230 \mathrm{~A}$ & {$[8]\left(n^{\circ} 22\right)$} \\
\hline $\mathrm{AP} / 58-2-37831$ & Clavicule gauche & 68 & $26230 \mathrm{~A}$ & [8] $\left(n^{\circ} 22\right)$ \\
\hline $\mathrm{AP} / 58-2-37863$ & Humérus droit & 68 & $26230 \mathrm{~A}$ & {$[8]\left(n^{\circ} 22\right)$} \\
\hline $\mathrm{AP} / 58-2-37862$ & Humérus gauche & 68 & $26230 \mathrm{~A}$ & [8] $\left(n^{\circ} 22\right)$ \\
\hline $\mathrm{AP} / 58-2-37864$ & Radius gauche & 68 & $26230 \mathrm{~A}$ & {$[8]\left(n^{\circ} 22\right)$} \\
\hline $\mathrm{AP} / 58-2-37865$ & Ulna gauche & 68 & $26230 \mathrm{~A}$ & [8] $\left(n^{\circ} 22\right)$ \\
\hline $\mathrm{AP} / 58-2-37886$ & Scaphoïde gauche & 69 & $26230 \mathrm{~A}$ & {$[8]\left(n^{\circ} 22\right)$} \\
\hline $\mathrm{AP} / 58-2-37885$ & Lunatum gauche & 69 & $26230 \mathrm{~A}$ & {$[8]\left(n^{\circ} 22\right)$} \\
\hline $\mathrm{AP} / 58-2-37887$ & Trapèze gauche & 69 & $26230 \mathrm{~A}$ & [8] $\left(n^{\circ} 22\right)$ \\
\hline AP/58-2-37888 & Trapézoïde gauche & 69 & $26230 \mathrm{~A}$ & {$[8]\left(n^{\circ} 22\right)$} \\
\hline $\mathrm{AP} / 58-2-37889$ & Triquetrum gauche & 69 & $26230 \mathrm{~A}$ & [8] $\left(n^{\circ} 22\right)$ \\
\hline $\mathrm{AP} / 58-2-37884$ & Capitatum gauche & 69 & $26230 \mathrm{~A}$ & {$[8]\left(n^{\circ} 22\right)$} \\
\hline $\mathrm{AP} / 58-2-37866$ & Hamatum gauche & 69 & $26230 \mathrm{~A}$ & [8] $\left(n^{\circ} 22\right)$ \\
\hline $\mathrm{AP} / 58-2-37890$ & MTC gauche 1 & 69 & $26230 \mathrm{~A}$ & {$[8]\left(n^{\circ} 22\right)$} \\
\hline $\mathrm{AP} / 58-2-37880$ & MTC gauche 2 & 69 & $26230 \mathrm{~A}$ & {$[8]\left(n^{\circ} 22\right)$} \\
\hline $\mathrm{AP} / 58-2-37881$ & MTC gauche 3 & 69 & $26230 \mathrm{~A}$ & [8] $\left(\mathrm{n}^{\circ} 22\right)$ \\
\hline $\mathrm{AP} / 58-2-37882$ & MTC gauche 4 & 69 & $26230 \mathrm{~A}$ & {$[8]\left(n^{\circ} 22\right)$} \\
\hline $\mathrm{AP} / 58-2-37883$ & MTC gauche 5 & 69 & $26230 \mathrm{~A}$ & [8] $\left(n^{\circ} 22\right)$ \\
\hline $\mathrm{AP} / 58-2-37874$ & Phalange prox. gauche 2 Main & 69 & $26230 \mathrm{~A}$ & {$[8]\left(n^{\circ} 22\right)$} \\
\hline $\mathrm{AP} / 58-2-37875$ & Phalange prox. gauche 3 Main & 69 & $26230 \mathrm{~A}$ & {$[8]\left(n^{\circ} 22\right)$} \\
\hline $\mathrm{AP} / 58-2-37876$ & Phalange prox. gauche 4 Main & 69 & $26230 \mathrm{~A}$ & {$[8]\left(n^{\circ} 22\right)$} \\
\hline $\mathrm{AP} / 58-2-37877$ & Phalange prox. gauche 5 Main & 69 & $26230 \mathrm{~A}$ & \\
\hline $\mathrm{AP} / 58-2-37870$ & Phalange méd. gauche 2 Main & 69 & $26230 \mathrm{~A}$ & \\
\hline $\mathrm{AP} / 58-2-37871$ & Phalange méd. gauche 3 Main & 69 & $26230 \mathrm{~A}$ & \\
\hline $\mathrm{AP} / 58-2-37872$ & Phalange méd. gauche 4 Main & 69 & $26230 \mathrm{~A}$ & \\
\hline $\mathrm{AP} / 58-2-37873$ & Phalange méd. gauche 5 Main & 69 & $26230 \mathrm{~A}$ & \\
\hline $\mathrm{AP} / 58-2-37867$ & Phalange dist. gauche 1 Main & 69 & $26230 \mathrm{~A}$ & \\
\hline $\mathrm{AP} / 58-2-37868$ & Phalange dist. gauche 3 Main & 69 & $26230 \mathrm{~A}$ & \\
\hline $\mathrm{AP} / 58-2-37869$ & Phalange dist. gauche 4 Main & 69 & $26230 \mathrm{~A}$ & \\
\hline
\end{tabular}


Tableau 18 Vestiges humains attribués à P4 d'après [6] et cette étude. Cf. Légende du tableau 15 / Human remains attributed to P4 after [6] and the present study. Cf. caption of table 15.

\begin{tabular}{|c|c|c|c|c|}
\hline $\mathbf{N}^{\circ}$ & Vestiges & $\mathbf{N}^{\circ}$ Boite MH & $\mathbf{N}^{\circ} \mathbf{M H}$ & Description bio. \\
\hline $\mathrm{AP} / 58-2-37856$ & Voûte (88 fragments) & 65 & $26230 \mathrm{~B}$ & [8] $\left(\right.$ Sans n $\left.{ }^{\circ}\right)$ \\
\hline $\mathrm{AP} / 58-2-37853$ & Voûte & 65 & $26230 \mathrm{~B}$ & [8] $\left(\right.$ Sans n $\left.{ }^{\circ}\right)$ \\
\hline $\mathrm{AP} / 58-2-37854$ & Voûte & 65 & $26230 \mathrm{~B}$ & [8] $\left(\right.$ Sans $\left.n^{\circ}\right)$ \\
\hline $\mathrm{AP} / 58-2-37855$ & Voûte & 65 & $26230 \mathrm{~B}$ & [8] $\left(\right.$ Sans n $\left.{ }^{\circ}\right)$ \\
\hline $\mathrm{AP} / 58-2-37833$ & Frontal & 65 & $26230 \mathrm{~B}$ & [8] $\left(\right.$ Sans $\left.n^{\circ}\right)$ \\
\hline $\mathrm{AP} / 58-2-37834$ & Frontal & 65 & $26230 \mathrm{~B}$ & [8] $\left(\right.$ Sans n $\left.{ }^{\circ}\right)$ \\
\hline $\mathrm{AP} / 58-2-37835$ & Frontal & 65 & $26230 \mathrm{~B}$ & [8] $\left(\right.$ Sans $\left.n^{\circ}\right)$ \\
\hline $\mathrm{AP} / 58-2-37837$ & Frontal droit & 65 & $26230 \mathrm{~B}$ & [8] $\left(\right.$ Sans n $\left.{ }^{\circ}\right)$ \\
\hline $\mathrm{AP} / 58-2-37836$ & Frontal gauche & 65 & $26230 \mathrm{~B}$ & [8] $\left(\right.$ Sans $\left.n^{\circ}\right)$ \\
\hline AP/58-2-37842 & Pariétal & 65 & $26230 \mathrm{~B}$ & [8] $\left(\right.$ Sans $\left.n^{\circ}\right)$ \\
\hline $\mathrm{AP} / 58-2-37843$ & Pariétal & 65 & $26230 \mathrm{~B}$ & [8] $\left(\right.$ Sans $\left.n^{\circ}\right)$ \\
\hline $\mathrm{AP} / 58-2-37845$ & Pariétal & 65 & $26230 \mathrm{~B}$ & [8] $\left(\right.$ Sans $\left.n^{\circ}\right)$ \\
\hline $\mathrm{AP} / 58-2-37846$ & Pariétal & 65 & 26230B & [8] $\left(\right.$ Sans $\left.n^{\circ}\right)$ \\
\hline $\mathrm{AP} / 58-2-37847$ & Pariétal & 65 & $26230 \mathrm{~B}$ & [8] $\left(\right.$ Sans n $\left.{ }^{\circ}\right)$ \\
\hline AP/58-2-37844 & Pariétal & 65 & $26230 \mathrm{~B}$ & [8] $\left(\right.$ Sans $\left.n^{\circ}\right)$ \\
\hline AP/58-2-37841 & Occipital & 65 & $26230 \mathrm{~B}$ & [8] $\left(\right.$ Sans $\left.n^{\circ}\right)$ \\
\hline $\mathrm{AP} / 58-2-37839$ & Occipital (2 fragments) & 11 & 25236 & [8] $\left(\right.$ Sans $\left.n^{\circ}\right)$ \\
\hline $\mathrm{AP} / 58-2-37840$ & Occipital (2 fragments) & 11 & 25236 & {$[8]\left(\right.$ Sans $\left.n^{\circ}\right)$} \\
\hline $\mathrm{AP} / 58-2-37838$ & Occipital & 65 & $26230 \mathrm{~B}$ & [8] $\left(\right.$ Sans $\left.n^{\circ}\right)$ \\
\hline $\mathrm{AP} / 58-2-37850$ & Temporal droit & 65 & $26230 \mathrm{~B}$ & [8] $\left(\right.$ Sans $\left.n^{\circ}\right)$ \\
\hline $\mathrm{AP} / 58-2-37851$ & Temporal gauche & 65 & $26230 \mathrm{~B}$ & [8] $\left(\right.$ Sans $\left.n^{\circ}\right)$ \\
\hline $\mathrm{AP} / 58-2-37852$ & Temporal gauche & 65 & $26230 \mathrm{~B}$ & [8] $\left(\right.$ Sans $\left.n^{\circ}\right)$ \\
\hline $\mathrm{AP} / 58-2-37849$ & Sphénoïde & 11 & 25236 & [8] $\left(\right.$ Sans $\left.n^{\circ}\right)$ \\
\hline AP/58-2-37848 & Sphénoïde & 11 & 25236 & [8] $\left(\right.$ Sans $\left.n^{\circ}\right)$ \\
\hline AP/58-2-37829 & Mandibule droite & 65 & $26230 \mathrm{~B}$ & [11] (“second sujet”) \\
\hline $\mathrm{AP} / 58-2-37861$ & Molaire déciduale 2 Inf droite & 65 & $26230 \mathrm{~B}$ & [11] ("second sujet") \\
\hline $\mathrm{AP} / 58-2-37860$ & Molaire déciduale 2 Inf gauche & 65 & $26230 \mathrm{~B}$ & [11] ("second sujet") \\
\hline $\mathrm{AP} / 58-2-37859$ & Molaire déciduale 2 Sup gauche & 65 & $26230 \mathrm{~B}$ & [11] (“second sujet”) \\
\hline $\mathrm{AP} / 58-2-37933$ & Vertèbre Indet. (11 fragments) & 65 & $26230 \mathrm{~B}$ & [8] (“17 vertèbres”) \\
\hline AP/58-2-37916 & Vertèbre Cervicale & 65 & $26230 \mathrm{~B}$ & [8] (“17 vertèbres") \\
\hline $\mathrm{AP} / 58-2-37926$ & Vertèbre Cervicale & 65 & 26230B & [8] (“17 vertèbres") \\
\hline $\mathrm{AP} / 58-2-37927$ & Vertèbre Cervicale & 65 & $26230 \mathrm{~B}$ & [8] (“17 vertèbres") \\
\hline AP/58-2-37928 & Vertèbre Cervicale & 65 & $26230 \mathrm{~B}$ & [8] (“17 vertèbres") \\
\hline $\mathrm{AP} / 58-2-37930$ & Vertèbre Cervicale & 65 & $26230 \mathrm{~B}$ & [8] (“17 vertèbres") \\
\hline AP/58-2-37925 & Vertèbre Cervicale & 65 & $26230 \mathrm{~B}$ & [8] (“17 vertèbres") \\
\hline AP/58-2-37929 & Vertèbre Cervicale & 65 & $26230 \mathrm{~B}$ & [8] (“17 vertèbres") \\
\hline AP/58-2-37931 & Vertèbre Cervicale & 65 & $26230 \mathrm{~B}$ & [8] (“17 vertèbres”) \\
\hline AP/58-2-37943 & Vertèbre Cervicale 1 & 65 & $26230 \mathrm{~B}$ & [8] (“17 vertèbres") \\
\hline AP/58-2-37944 & Vertèbre Cervicale 2 & 65 & $26230 \mathrm{~B}$ & [8] (“17 vertèbres”) \\
\hline $\mathrm{AP} / 58-2-37940$ & Vertèbre Cervicale 3 à 7 & 65 & $26230 \mathrm{~B}$ & [8] (“17 vertèbres") \\
\hline AP/58-2-37932 & Vertèbre Cervicale (7) ou Thoracique (1) & 65 & 26230B & [8] (“17 vertèbres") \\
\hline AP/58-2-37894 & Vertèbre Thoracique & 65 & 26230B & [8] (“17 vertèbres") \\
\hline $\mathrm{AP} / 58-2-37895$ & Vertèbre Thoracique & 65 & $26230 \mathrm{~B}$ & [8] (“17 vertèbres") \\
\hline $\mathrm{AP} / 58-2-37917$ & Vertèbre Thoracique & 65 & $26230 \mathrm{~B}$ & [8] ("17 vertèbres") \\
\hline $\mathrm{AP} / 58-2-37935$ & Vertèbre Thoracique & 65 & $26230 \mathrm{~B}$ & [8] (“17 vertèbres”) \\
\hline $\mathrm{AP} / 58-2-37936$ & Vertèbre Thoracique & 65 & 26230B & $\begin{array}{l}\text { [8] ("17 vertèbres") } \\
\text { (Suite page suivante) }\end{array}$ \\
\hline
\end{tabular}




\begin{tabular}{|c|c|c|c|c|}
\hline $\mathbf{N}^{\circ}$ & Vestiges & $\mathbf{N}^{\circ}$ Boite MH & $\mathbf{N}^{\circ} \mathbf{M H}$ & Description bio. \\
\hline AP/58-2-37937 & Vertèbre Thoracique & 65 & $26230 \mathrm{~B}$ & [8] (“17 vertèbres”) \\
\hline $\mathrm{AP} / 58-2-37896$ & Vertèbre Thoracique & 65 & 26230B & [8] (“17 vertèbres") \\
\hline AP/58-2-37914 & Vertèbre Thoracique & 65 & $26230 \mathrm{~B}$ & [8] (“17 vertèbres”) \\
\hline $\mathrm{AP} / 58-2-37904$ & Vertèbre Thoracique & 65 & $26230 \mathrm{~B}$ & [8] (“17 vertèbres”) \\
\hline $\mathrm{AP} / 58-2-37939$ & Vertèbre Thoracique & 65 & $26230 \mathrm{~B}$ & [8] (“17 vertèbres”) \\
\hline $\mathrm{AP} / 58-2-37897$ & Vertèbre Thoracique & 65 & $26230 \mathrm{~B}$ & [8] (“17 vertèbres”) \\
\hline $\mathrm{AP} / 58-2-37913$ & Vertèbre Thoracique & 65 & $26230 \mathrm{~B}$ & [8] (“17 vertèbres”) \\
\hline AP/58-2-37938 & Vertèbre Thoracique & 65 & $26230 \mathrm{~B}$ & [8] (“17 vertèbres”) \\
\hline $\mathrm{AP} / 58-2-37906$ & $\begin{array}{l}\text { Vertèbre Thoracique (11/12) } \\
\text { ou Lombaire (1) }\end{array}$ & 65 & 26230B & [8] (“17 vertèbres”) \\
\hline $\mathrm{AP} / 58-2-37934$ & Vertèbre Lombaire ( 6 fragments) & 65 & $26230 \mathrm{~B}$ & [8] (“17 vertèbres”) \\
\hline $\mathrm{AP} / 58-2-37915$ & Côte droite 1 & 65 & 26230B & [8] ("11 côtes droites") \\
\hline $\mathrm{AP} / 58-2-37945$ & Côte droite 2 & 65 & $26230 \mathrm{~B}$ & [8] ("11 côtes droites") \\
\hline $\mathrm{AP} / 58-2-37907$ & Côte droite 3 & 65 & $26230 \mathrm{~B}$ & [8] ("11 côtes droites") \\
\hline $\mathrm{AP} / 58-2-37905$ & Côte droite 4 & 65 & $26230 \mathrm{~B}$ & [8] ("11 côtes droites") \\
\hline AP/58-2-37918 & Côte droite 5 & 65 & $26230 \mathrm{~B}$ & [8] ("11 côtes droites") \\
\hline $\mathrm{AP} / 58-2-37921$ & Côte droite 6 & 65 & $26230 \mathrm{~B}$ & [8] ("11 côtes droites") \\
\hline AP/58-2-37908 & Côte droite 7 & 65 & 26230B & [8] ("11 côtes droites") \\
\hline $\mathrm{AP} / 58-2-37919$ & Côte droite 8 & 65 & $26230 \mathrm{~B}$ & [8] ("11 côtes droites") \\
\hline $\mathrm{AP} / 58-2-37920$ & Côte droite 9 & 65 & $26230 \mathrm{~B}$ & [8] ("11 côtes droites") \\
\hline $\mathrm{AP} / 58-2-37922$ & Côte droite 10 & 65 & $26230 \mathrm{~B}$ & [8] ("11 côtes droites") \\
\hline $\mathrm{AP} / 58-2-37816$ & Côte droite 11 & 65 & $26230 \mathrm{~B}$ & [8] ("11 côtes droites") \\
\hline $\mathrm{AP} / 58-2-37910$ & Côte droite 12 & 65 & $26230 \mathrm{~B}$ & [8] ("11 côtes droites") \\
\hline $\mathrm{AP} / 58-2-37902$ & Côte gauche 1 & 65 & $26230 \mathrm{~B}$ & [8] ("8 côtes gauches") \\
\hline AP/58-2-37903 & Côte gauche 2 & 65 & $26230 \mathrm{~B}$ & [8] ("8 côtes gauches") \\
\hline $\mathrm{AP} / 58-2-37923$ & Côte gauche 3 & 65 & $26230 \mathrm{~B}$ & [8] ("8 côtes gauches") \\
\hline $\mathrm{AP} / 58-2-37901$ & Côte gauche 4 & 65 & $26230 \mathrm{~B}$ & [8] ("8 côtes gauches") \\
\hline $\mathrm{AP} / 58-2-37924$ & Côte gauche 5 & 65 & $26230 \mathrm{~B}$ & [8] ("8 côtes gauches") \\
\hline AP/58-2-37898 & Côte gauche 6 & 65 & $26230 \mathrm{~B}$ & [8] ("8 côtes gauches") \\
\hline $\mathrm{AP} / 58-2-37893$ & Côte gauche 7 & 65 & $26230 \mathrm{~B}$ & [8] ("8 côtes gauches") \\
\hline $\mathrm{AP} / 58-2-37912$ & Côte gauche 8 & 65 & $26230 \mathrm{~B}$ & [8] ("8 côtes gauches") \\
\hline $\mathrm{AP} / 58-2-37911$ & Côte gauche 9 & 65 & $26230 \mathrm{~B}$ & [8] ("8 côtes gauches") \\
\hline $\mathrm{AP} / 58-2-37900$ & Côte gauche 10 & 65 & $26230 \mathrm{~B}$ & [8] ("8 côtes gauches") \\
\hline AP/58-2-37899 & Côte gauche 11 & 65 & $26230 \mathrm{~B}$ & [8] ("8 côtes gauches") \\
\hline $\mathrm{AP} / 58-2-37809$ & Côte gauche 12 & 65 & $26230 \mathrm{~B}$ & [8] ("8 côtes gauches") \\
\hline $\mathrm{AP} / 58-2-37941$ & Clavicule droite & 65 & $26230 \mathrm{~B}$ & {$[8]\left(\right.$ Sans n $\left.^{\circ}\right)$} \\
\hline $\mathrm{AP} / 58-2-37942$ & Clavicule gauche & 65 & $26230 \mathrm{~B}$ & {$[8]\left(\right.$ Sans $\left.n^{\circ}\right)$} \\
\hline $\mathrm{AP} / 58-2-37879$ & MTC 2,3 ou 4 & 65 & & \\
\hline
\end{tabular}


Tableau 19 Vestiges humains attribués à P5 d'après [6] et cette étude. Cf. Légende du tableau 15 / Human remains attributed to P5 after [6] and the present study. Cf. caption of table 15.

\begin{tabular}{|lllll|}
\hline $\mathbf{N}^{\circ}$ & Vestiges & $\mathbf{N}^{\circ}$ Boite MH & $\mathbf{N}^{\circ} \mathbf{M H}$ & Description bio. \\
\hline AP/58-2-2F592 & Scapula Gauche & & Cette étude \\
AP/58-2-F1026A & Clavicule Gauche & & Cette étude \\
AP/58-2-F1026B & Clavicule Droite & 64 & & Cette étude \\
AP/58-2-37805 & Humérus gauche & 64 & 26231 & {$[8]\left(\mathrm{n}^{\circ} 1\right)$} \\
AP/58-2-37814 & Radius gauche (2 fragments) & 64 & 26231 & {$[8]\left(\mathrm{n}^{\circ} 7\right)$} \\
AP/58-2-37813 & Ulna gauche & & 26231 & {$[8]\left(\mathrm{n}^{\circ} 7\right)$} \\
AP/58-2-37964 & MTC Droit 3 & & Cette étude \\
\hline
\end{tabular}

Tableau 20 Vestiges humains attribués à P6 d'après [6] et cette étude. Cf. Légende du tableau 15 / Human remains attributed to P6 after [6] and the present study. Cf. caption of table 15.

\begin{tabular}{|lllll|}
\hline $\mathbf{N}^{\circ}$ & Vestiges & $\mathbf{N}^{\circ}$ Boite MH & $\mathbf{N}^{\circ}$ MH & Description bio. \\
\hline AP/58-2-37857 & Frontal & 11 & 25236 & {$[8]\left(\mathrm{pas} \mathrm{de} \mathrm{n}^{\circ}\right)$} \\
AP/58-2-37812 & Temporal gauche & 11 & 25236 & {$[8]\left(\mathrm{n}^{\circ} 6\right)$} \\
AP/58-2-37818 & Zygomatique droit & 11 & 25236 & {$[8]\left(\mathrm{n}^{\circ} 11\right)$} \\
AP/58-2-37821 & Maxillaire droit et dents & 11 & 25236 & {$[8]\left(\mathrm{n}^{\circ} 14\right)$ et $[11]$} \\
& & & ("troisième sujet") \\
AP/58-2-F2283 & Canine déciduale Sup droite & & Cette étude \\
AP/07-2-W76A-T550 & Incisive déciduale 2 Sup gauche & & Cette étude \\
AP/58-2-37819 & Molaire 1 Sup droite & 11 & 25236 & {$[11]$ ("troisième sujet") } \\
AP/58-2-37956 & Vertèbre Cervicale 1 & & Cette étude \\
AP/58-2-37957 & Vertèbre Cervicale 2 & & Cette étude \\
AP/58-2-37952 & Scapula droite & & Cette étude \\
AP/58-2-37959 & Clavicule gauche & & Cette étude \\
\hline
\end{tabular}

\section{Références}

1. Chiotti L, Nespoulet R, Henry-Gambier D (2013) Une nouvelle fouille à l'abri Pataud. In: Nespoulet R, Chiotti L, HenryGambier D (eds) Le Gravettien final de l'abri Pataud (Dordogne, France). Fouilles et études 2005-2009. Archaeopress, BAR International Series, 2458, Oxford, pp 13-21

2. Henry-Gambier D, Nespoulet R, Chiotti L, et al (2013) Datations. In: Nespoulet R, Chiotti L, Henry-Gambier D (eds) Le Gravettien final de l'abri Pataud (Dordogne, France). Fouilles et études 2005-2009. Archaeopress, BAR International Series, 2458, Oxford, pp 43-50

3. Nespoulet R, Chiotti L, Henry-Gambier D (2013) Synthèse. In: Nespoulet R, Chiotti L, Henry-Gambier D (eds) Le Gravettien final de l'abri Pataud (Dordogne, France). Fouilles et études 2005-2009. Archaeopress, BAR International Series, 2458, Oxford, pp 179-86

4. Chiotti L, Nespoulet R, Henry-Gambier D (sous presse) Occupations and status of the Abri Pataud (Dordogne, France) during the Final Gravettian. Quaternary International

5. Chiotti L, Nespoulet R, Henry-Gambier D, et al (2009) Statut des objets « extra-ordinaires » du Gravettien final de l'abri Pataud (Les Eyzies-de-Tayac, Dordogne) : objets abandonnés dans l'ha- bitat ou dépôt intentionnel ? In: Bonnardin S, Hamon C, Lauwers M, Quilliec B (eds) Du matériel au spirituel. Réalités archéologiques et historiques des "dépôts " de la Préhistoire à nos jours. XXIXe rencontres internationales d'archéologie et d'histoire d'Antibes. Éditions APDCA, Antibes, pp 305-22

6. Henry-Gambier D, Villotte S, Beauval C, et al (2013) Les vestiges humains : un assemblage original. In: Nespoulet R, Chiotti L, Henry-Gambier D (eds) Le Gravettien final de l'abri Pataud (Dordogne, France). Fouilles et études 2005-2009. Archaeopress, BAR International Series, 2458, Oxford, pp 135-77

7. Chiotti L, Nespoulet R, Henry-Gambier D, et al (2014) Un comportement funéraire original au Gravettien Final. Bilan des analyses et études 2005-2011 de la couche 2 de l'abri Pataud (Les Eyzies-de-Tayac, Dordogne). In: Paillet P (ed) Les arts de la Préhistoire : micro-analyses, mises en contexte et conservation, actes du colloque MADAPCA, 16-18 novembre 2011. Paléo, numéro spécial, pp 183-93

8. Billy G (1975) Etude anthropologique des restes humains de l'Abri Pataud. In: Movius Jr H L (ed) Excavation of the Abri Pataud. Les Eyzies (Dordogne): Contributors. Harvard University, Peabody Museum, American School of Prehistoric Research, bull. 30, Cambridge, pp 201-61

9. Legoux P (1972) Étude odontologique des restes humains périgordiens et protomagdaléniens de l'Abri Pataud (Dordogne). 
Bulletins et Mémoires de la Société d'anthropologie de Paris, XII ${ }^{\circ}$ Série 9(4):293-330

10. Legoux P (1974) Étude odontologique des restes humains périgordiens et proto-magdaléniens de l'Abri-Pataud (Dordogne) (seconde partie). Bulletins et Mémoires de la Société d'anthropologie de Paris, XIII ${ }^{\circ}$ Série 1(1):45-84

11. Legoux P (1975) Présentation des dents des restes humains de l'Abri Pataud. In: Movius Jr H L (ed) Excavation of the Abri Pataud. Les Eyzies (Dordogne): Contributors. Harvard University, Peabody Museum, American School of Prehistoric Research, bull. 30, Cambridge, pp 262-304

12. Villotte S, Brůžek J, Henry-Gambier D (2011) Caractéristiques biologiques des sujets adultes gravettiens : révision de l'âge au décès et du sexe. In: Goutas N, Guillermin P, Klaric L, Pesesse D (eds) Á la recherche des identités gravettiennes : actualités, questionnements et perspectives, Actes de la table ronde sur le Gravettien en France et dans les pays limitrophes. Mémoires de la Société Préhistorique Française, 52 pp 209-16

13. Bräuer G (1988) Osteometrie. In: Knussmann R (ed) Anthropologie: handbuch der vergleichenden Biologie des Menschen. G. Fischer, Stuttgart, pp 160-232

14. Sládek V, Trinkaus E, Hillson SW, et al (2000) The people of the Pavlovian. Skeletal catalogue and osteometrics of the Gravettian fossil hominids from Dolní Věstonice and Pavlov, Academy of Sciences of the Czech Republic, Brno, $244 \mathrm{p}$

15. Smith BH (1984) Patterns of molar wear in hunter-gatherers and agriculturalists. Am J Phys Anthropol 63(1):39-56

16. Turner CG, Nichol CR, Scott GR (1991) Scoring procedures for key morphological traits of the permanent dentition: The Arizona State University Dental Anthropology System. In: Kelley M A, Larsen C S (eds) Advances in dental anthropology. Wiley-Liss, New York, pp 13-31

17. Formicola V (1988) The male and the female in the Upper Paleolithic burials from Grimaldi Caves (Liguria, Italy). Bulletin du Musée d'Anthropologie Préhistorique de Monaco 31:41-48

18. Vallois HV, Billy G (1965) Nouvelles recherches sur les hommes fossiles de l'abri de Cro-Magnon. L'Anthropologie 69(1-2):47-74

19. Gambier D, Bruzek J, Schmitt A, et al (2006) Révision du sexe et de l'âge au décès des fossiles de Cro-Magnon (Dordogne, France) à partir de l'os coxal. C. R. Palevol 5:735-41

20. Trinkaus E, Svoboda J (2006) Early modern human evolution in Central Europe. The people of Dolní Věstonice and Pavlov, Oxford University Press, New York, $489 \mathrm{p}$

21. Verneau R (1906) Les grottes de Grimaldi (Baoussé-Roussé), Anthropologie, II-1, Imprimerie de Monaco, Monaco, $212 \mathrm{p}$

22. Mallegni F, Palma di Cesnola A (1994) Les restes hunains découverts dans les niveaux gravettiens de la grotte Paglicci (Rignano Garganico, Pouilles, Italie). anthropologie (Brno) 32(1):45-57

23. Trinkaus E (2000) Late Pleistocene and Holocene human remains from Paviland Cave. In: Aldhous-Green S (ed) Paviland Cave and the "Red Lady". A definitive report. Western Academic \& Specialist Press Limited, Bristol, pp 141-99

24. Mallegni F, Bertoldi F, Manolis S (2000) Palaeobiology of two Gravettian skeletons from Veneri cave (Parabita, Puglia, Italy). Homo 51(2-3):235-57

25. Trinkaus E, Buzhilova AP, Mednikova MB, et al (2014) The people of Sunghir. burials, bodies, and behavior in the Earlier Upper Paleolithic, Oxford University Press, New York, $368 \mathrm{p}$

26. Sergi S, Parenti R, Paoli G (1974) Il giovane paleolitico della Caverna delle Arene Candide. Studi di Paletnologia, Paleoantropologia, Paleontologia e Geologia del Quaternario 2:13-38

27. Hillson SW, Trinkaus E (2002) Comparative dental crown metrics. In: Zilhao J, Trinkaus E (eds) Portrait of the artist as a child. The Gravettian human skeleton from the Abrigo do Lagar Velho and its archaeological context. Trabalhos de Arqueologia 22, Lisboa, pp 356-64

28. Mallegni F, Parenti R (1972-1973) Studio antropologico di uno scheletro giovanile d'epoca gravettiana raccolto nella grotta Paglicci (Rignano Garganico). Rivista di Antropologia LVIII:317-42

29. Matiegka J (1934) Homo předmostensis, fosilní člověk z Předmostí na Moravě. T1, Nákladem České akademie věd a umění, $145 \mathrm{p}$

30. Martínez I, Arsuaga JL, Quam R, et al (2008) Human hyoid bones from the middle Pleistocene site of the Sima de los Huesos (Sierra de Atapuerca, Spain). J Hum Evol 54(1):118-24

31. Hauser G, De Stefano GF (1989) Epigenetic variants of the human skull, Schweizerbart'sche Verlagsbuchhandlung, Stuttgart, $301 \mathrm{p}$

32. Crubézy E, Telmon N, Sevin A, et al (1999) Microévolution d'une population historique. Étude des caractères discrets de la populationde Missiminia (Soudan, IIIe-VIe siècle). Bulletins et Mémoires de la Société d'Anthropologie de Paris, n.s., 11(1):1-213

33. Trinkaus E (2006) The upper limb remains. In: Trinkaus E, Svoboda $\mathrm{J}$ (eds) Early modern human evolution in Central Europe. The people of Dolní Věstonice and Pavlov. Oxford University Press, New York, pp 327-372

34. Trinkaus E (2006) The lower limb remains. In: Trinkaus E, Svoboda J (eds) Early modern human evolution in Central Europe. The people of Dolní Věstonice and Pavlov. Oxford University Press, New York, pp 380-418

35. AlQahtani SJ, Hector MP, Liversidge HM (2010) Brief communication: The London atlas of human tooth development and eruption. Am J Phys Anthropol 142(3):481-490.

36. Scheuer L, Black S (2000) Developmental juvenile osteology, Academic Press, San Diego, 587 p

37. Villotte S, Knüsel CJ (2013) Understanding entheseal changes: Definition and life course changes. International Journal of Osteoarchaeology 23(2):135-46

38. Holt BM, Formicola V (2008) Hunters of the Ice Age: The biology of Upper Paleolithic people. Am J Phys Anthropol 137 (S47):70-99 